DESY 09-078

PITHA 09/11

\title{
Soft gluon resummation for the production of gluino-gluino and squark-antisquark pairs at the LHC
}

\author{
A. KuleszA ${ }^{a}$ And L. MOtYKA ${ }^{b, c} \dagger$ \\ ${ }^{a}$ Institut für Theoretische Physik E, RWTH Aachen, D-52056 Aachen, Germany \\ ${ }^{b}$ II Institute for Theoretical Physics, University of Hamburg, \\ Luruper Chaussee 149, D-22761, Germany \\ ${ }^{c}$ Institute of Physics, Jagellonian University, Reymonta 4, \\ 30-059 Kraków, Poland
}

\begin{abstract}
We study the effect of soft gluon emission in the hadroproduction of gluino-gluino and squarkantisquark pairs at the next-to-leading logarithmic (NLL) accuracy within the framework of the minimal supersymmetric model. We present the calculation of the one-loop soft anomalous dimension matrices controlling the colour evolution of the underlying hard-scattering processes. The numerical results for resummed cross sections for proton-proton collisions at the Large Hadron Collider are discussed in detail.
\end{abstract}

\footnotetext{
†anna.kulesza@physik.rwth-aachen.de, leszek.motyka@desy.de
} 


\section{Introduction}

Supersymmetry (SUSY) is one of the most promising candidates for the theory of physics beyond the Standard Model (SM). In the coming years, experiments at the Large Hadron Collider (LHC) will undertake searches for new physical phenomena. A large part of this effort will be devoted to looking for signals of SUSY.

One of the most studied SUSY models is the Minimal Supersymmetric Standard Model (MSSM) [1], characterized by the minimal content of supersymmetric particles and $R$-parity conservation. Within the MSSM, the dominant production processes of sparticles at the LHC are those involving pairs of coloured particles, i.e. squarks and gluinos, in the final state [2]. The exact discovery reach of the LHC is model-dependent but it is expected that the discovery of squarks and gluinos should be possible for masses of up to around $2 \mathrm{TeV}$ [3]. Since the hadroproduction cross sections for strongly-interacting sparticles depend only on the masses of squarks and gluinos $[4,5,6,7]$, measurements of total cross sections for coloured sparticle production may be used to determine values of the fundamental MSSM parameters, e.g. the masses of sparticles $[4,6,7,8]$, or to draw exclusion limits for the mass parameters $[9,10]$. The precision of the mass determination (exclusion limit) will crucially depend on the accuracy of the corresponding theoretical predictions. With the large production rates expected at the LHC it is thus of utter importance to study the total cross sections for the hadroproduction of squarks and gluinos with the highest possible theoretical accuracy.

The leading-order $(\mathrm{LO})$ total cross sections of $\mathcal{O}\left(\alpha_{\mathrm{s}}^{2}\right)$ were calculated long time ago $[4,5]$. The corresponding next-to-leading order (NLO) SUSY-QCD corrections are known for all hadroproduction processes of pairs of squarks and gluinos $[6,7]$. The NLO electroweak (EW) corrections [11] for the processes involving squarks in the final state are also known, as well as the LO EW $\mathcal{O}\left(\alpha^{2}\right)$ total cross sections and the $\mathcal{O}\left(\alpha \alpha_{\mathrm{s}}\right)$ LO EW-QCD interference predictions [11, 12].

The NLO SUSY-QCD corrections have been found to be positive and large. Among the pairproduction processes of coloured sparticles at the LHC, the gluino-pair $(\tilde{g} \tilde{g})$ production receives the largest NLO SUSY-QCD correction that may reach $100 \%$ for gluino mass $m_{\tilde{g}}=1 \mathrm{TeV}$ and $1.2 \mathrm{TeV} \lesssim m_{\tilde{q}} \lesssim 2 \mathrm{TeV}[7]$. The corrections to the squark-antisquark ( $\left.\tilde{q} \overline{\tilde{q}}\right)$ total cross section can be also sizable, of order of $30 \%$ for the squark mass $m_{\tilde{q}}=1 \mathrm{TeV}$, and are the second largest in a certain range of mass parameters. The occurrence of large corrections indicates that computation of higher order SUSY-QCD corrections is necessary in order to achieve precise theoretical predictions.

A large part of the NLO SUSY-QCD corrections to the total cross section for $\tilde{g} \tilde{g}$ and $\tilde{q} \overline{\tilde{q}}$ processes comes from production close to threshold [7]. The threshold region is reached when the square of the partonic center-of-mass (c.o.m.) energy, $\hat{s}$, approaches $4 m^{2}$, where $m$ is the average particle mass in the produced pair. The velocity of the produced heavy particles in 
the partonic c.o.m. system $\beta \equiv \sqrt{1-4 m^{2} / \hat{s}}$ is then small, $\beta \ll 1$. In this region two types of corrections dominate: Coulomb corrections due to exchange of gluons between slowly moving massive particles and soft gluon corrections due to emission of low energy gluons off the coloured initial and final states. The soft gluon corrections are enhanced by powers of large logarithms of $\beta$, i.e. at the NLO one finds terms in the relative corrections proportional to $\alpha_{\mathrm{s}} \log ^{2}\left(\beta^{2}\right)$ that become sizeable when $\beta^{2} \sim \exp \left(-1 / \sqrt{\alpha_{\mathrm{s}}}\right)$. At the $n$-th order of the perturbative expansion in the strong coupling $\alpha_{\mathrm{s}}$ the total cross sections receive corrections proportional to $\alpha_{\mathrm{s}}^{n} \log ^{k}\left(\beta^{2}\right)$ where $k=2 n, \ldots, 0$. Sufficiently close to the partonic threshold fixed-order expressions for the cross sections are bound to fail. However, the logarithmic contributions can be taken into account to all orders in $\alpha_{\mathrm{s}}$ by means of threshold resummation. Resummed predictions are particularly important for processes with large masses in the final states since then the bulk of production comes from the threshold region. This is exactly the case for production of sparticles which are expected to be heavier than the SM particles. Additionally, if partonic subprocesses involve gluons in the initial state, the soft-gluon effects, and thus the impact of resummation, are expected to be significant due to the high colour charge of the gluons.

Crucially, calculation of the soft gluon corrections provides a reliable estimate of unknown higher order terms beyond the NLO, what results in reduction of the theoretical uncertainty due to scale variation. In a recent letter [13] we have presented results for threshold-resummed cross sections at the next-to-leading logarithm (NLL) accuracy for hadroproduction of $\tilde{g} \tilde{g}$ and $\tilde{q} \overline{\tilde{q}}$ pairs at the LHC. A dominant part of the next-to-next-to-leading (NNLO) correction for the $\tilde{q} \overline{\tilde{q}}$ production consisting of terms coming from the expansion of the resummed exponent at the next-to-next-to-leading logarithm (NNLL) level, Coulomb terms and the universal scale terms, was later calculated in [14]. Moreover, threshold resummation for single colour-octet scalar at the LHC was also studied [15]. Further work on resummation for production of coloured sparticle pairs is to be found in $[16,17]$.

The hadronic production processes of $\tilde{g} \tilde{g}$ and $\tilde{q} \overline{\tilde{q}}$ pairs are scattering processes with a nontrivial colour flow structure. At the NLL level resummation requires including contributions from soft gluons emitted at wide angles. Such emission is sensitive to the colour flow of the underlying hard scattering and the evolution of the colour exchange is governed by the soft anomalous dimension matrix $[18,19,20,21,22]$. The one-loop soft anomalous dimension matrices were first calculated for heavy-quark and dijet production [18, 19, 21, 23]. In [13] we have presented the explicit form of the one-loop soft anomalous dimension matrices for partonic subprocesses contributing to $\tilde{g} \tilde{g}$ hadroproduction. The general results for any $2 \rightarrow n$ QCD process with massless particles in the final state were derived at one- [22], and two-loops [24]. The twoloop anomalous dimension for the pair production of heavy quarks was also determined in the threshold limit [25], and employed later in [14]. Recently, the structure of the massive two-loop matrix for any $2 \rightarrow n$ process has been studied in [26]. 
In this paper we discuss in detail the derivation of the analytical results presented in [13] and carry out a thorough study of the numerical results as well as perform resummation of the leading Coulomb corrections for the $\tilde{q} \overline{\tilde{q}}$ and $\tilde{g} \tilde{g}$ production processes.

\section{Leading order results}

The hadronic cross section for the process $h_{1} h_{2} \rightarrow k l$ reads

$$
\sigma_{h_{1} h_{2} \rightarrow k l}\left(S,\left\{m^{2}\right\}\right)=\sum_{i, j} \int d x_{1} d x_{2} f_{i / h_{1}}\left(x_{1}, \mu_{F}\right) f_{j / h_{2}}\left(x_{2}, \mu_{F}\right) \hat{\sigma}_{i j \rightarrow k l}\left(\hat{s},\left\{m^{2}\right\}, \mu_{F}^{2}, \mu_{R}^{2}\right),
$$

where $S(\hat{s})$ is the square of the hadronic (partonic) center-of-mass energy and $\left\{m^{2}\right\}$ stands for all masses entering the calculations. The parton distribution functions (pdfs) $f_{i / h}$ are taken at the factorisation scale $\mu_{F}$. We set $\mu_{F}$ equal to the renormalisation scale $\mu_{R}$ in our calculations.

The expressions for the LO partonic cross sections $\hat{\sigma}_{i j \rightarrow k l}\left(s,\left\{m^{2}\right\}, \mu_{F}^{2}, \mu_{R}^{2}\right)$ for all squark and gluino hadroproduction processes can be found in [7]. Here we present the contributions to the LO cross sections for the $\tilde{q} \overline{\tilde{q}}$ and $\tilde{g} \tilde{g}$ production coming from different colour channels. For each partonic process we define the corresponding colour basis in the $s$-channel.

For the $\tilde{q} \overline{\tilde{q}}$ production we consider the processes

$$
q_{i}\left(p_{i}, \alpha_{i}\right) \bar{q}\left(p_{j}, \alpha_{j}\right) \rightarrow \tilde{q}\left(p_{k}, \alpha_{k}\right) \overline{\tilde{q}}\left(p_{l}, \alpha_{l}\right)
$$

and

$$
g\left(p_{i}, a_{i}\right) g\left(p_{j}, a_{j}\right) \rightarrow \tilde{q}\left(p_{k}, \alpha_{k}\right) \overline{\tilde{q}}\left(p_{l}, \alpha_{l}\right)
$$

where $p$ are particle four-momenta and $\alpha$ and $a$ are colour indices in the fundamental and adjoint representation of $\mathrm{SU}(3)$, correspondingly. In the quark-channel (2) we have only two possible colour exchanges: the singlet and the octet, $\{\mathbf{1}, \mathbf{8}\}$, and the basis consists of two colour tensors

$$
\begin{aligned}
c_{\mathbf{1}}^{q, \tilde{q}} & =\delta^{\alpha_{i} \alpha_{j}} \delta^{\alpha_{k} \alpha_{l}}, \\
c_{\mathbf{8}}^{q, \tilde{q}} & =-\frac{1}{6} \delta^{\alpha_{i} \alpha_{j}} \delta^{\alpha_{k} \alpha_{l}}+\frac{1}{2} \delta^{\alpha_{i} \alpha_{k}} \delta^{\alpha_{j} \alpha_{l}} .
\end{aligned}
$$

In the gluon channel, the basis is built out of three tensors corresponding to $\left\{\mathbf{1}_{\mathbf{1}} \mathbf{8}_{\mathbf{S}}, \mathbf{8}_{\mathbf{A}}\right\}$ representations

$$
\begin{gathered}
c_{\mathbf{1}}^{g, \tilde{q}}=\delta^{a_{i} a_{j}} \delta^{\alpha_{k} \alpha_{l}}, \\
c_{\mathbf{8}_{\mathbf{S}}}^{g, \tilde{q}}=T_{\alpha_{l} \alpha_{k}}^{b} d^{b a_{i} a_{j}}, \\
c_{\mathbf{8}_{\mathbf{A}}^{g, \tilde{q}}}^{g,}=i T_{\alpha_{l} \alpha_{k}}^{b} f^{b a_{i} a_{j}} .
\end{gathered}
$$

where $T^{b}$ matrices are the $\mathrm{SU}(3)$ generators. 
At the leading order, two partonic channels contribute to the $\tilde{g} \tilde{g}$ production:

$$
q\left(p_{i}, \alpha_{i}\right) \bar{q}\left(p_{j}, \alpha_{j}\right) \rightarrow \tilde{g}\left(p_{k}, a_{k}\right) \tilde{g}\left(p_{l}, a_{l}\right)
$$

and

$$
g\left(p_{i}, a_{i}\right) g\left(p_{j}, a_{j}\right) \rightarrow \tilde{g}\left(p_{k}, a_{k}\right) \tilde{g}\left(p_{l}, a_{l}\right) .
$$

For the process (6) the colour basis is the same as for the $\tilde{q} \overline{\tilde{q}}$ production in the gluon-channel (3) and is given by (5) after interchanging the indices $(i j) \leftrightarrow(k l)$. For the $g g$ channel there are eight independent colour tensors. Following [21] we choose an orthogonal basis, $\left\{c_{I}^{g, \tilde{g}}\right\}, I=1,2, \ldots, 8$, consisting of five tensors $c_{1}^{g, \tilde{g}}, c_{2}^{g, \tilde{g}}, c_{3}^{g, \tilde{g}}, c_{4}^{g, \tilde{g}}$ and $c_{5}^{g, \tilde{g}}$ corresponding to the $\mathbf{1}, \mathbf{8}_{\mathbf{S}}, \mathbf{8}_{\mathbf{A}}, \mathbf{1 0} \oplus \overline{\mathbf{1 0}}$ and $\mathbf{2 7}$ representations in the $s$-channel, and three additional tensors, $c_{6}^{g, \tilde{g}}, c_{7}^{g, \tilde{g}}$, and $c_{8}^{g, \tilde{g}}$. The base tensors are

$$
\begin{aligned}
c_{1}^{g, \tilde{g}} & =\frac{1}{8} \delta^{a_{i} a_{j}} \delta^{a_{k} a_{l}}, \\
c_{2}^{g, \tilde{g}} & =\frac{3}{5} d^{a_{i} a_{j} b} d^{b a_{k} a_{l}}, \\
c_{3}^{g, \tilde{g}} & =\frac{1}{3} f^{a_{i} a_{j} b} f^{b a_{k} a_{l}}, \\
c_{4}^{g, \tilde{g}} & =\frac{1}{2}\left(\delta^{a_{i} a_{k}} \delta^{a_{j} a_{l}}-\delta^{a_{i} a_{l}} \delta^{a_{j} a_{k}}\right)-\frac{1}{3} f^{a_{i} a_{j} b} f^{b a_{k} a_{l}}, \\
c_{5}^{g, \tilde{g}} & =\frac{1}{2}\left(\delta^{a_{i} a_{k}} \delta^{a_{j} a_{l}}+\delta^{a_{i} a_{l}} \delta^{a_{j} a_{k}}\right)-\frac{1}{8} \delta^{a_{i} a_{j}} \delta^{a_{k} a_{l}} \\
& -\frac{3}{5} d^{a_{i} a_{j} b} d^{b a_{k} a_{l}}, \\
c_{6}^{g, \tilde{g}} & =\frac{i}{4}\left(f^{a_{i} a_{j} b} d^{b a_{k} a_{l}}+d^{a_{i} a_{j} b} f^{b a_{k} a_{l}}\right), \\
c_{7}^{g, \tilde{g}} & =\frac{i}{4}\left(f^{a_{i} a_{j} b} d^{b a_{k} a_{l}}-d^{a_{i} a_{j} b} f^{b a_{k} a_{l}}\right), \\
c_{8}^{g, \tilde{g}} & =\frac{i}{4}\left(d^{a_{i} a_{k} b} f^{b a_{j} a_{l}}+f^{a_{i} a_{k} b} d^{b a_{j} a_{l}}\right) .
\end{aligned}
$$

In the set of basis defined above, we obtain the following colour-channel contributions to the total cross section for $q_{i} \bar{q}_{j} \rightarrow \tilde{q} \overline{\tilde{q}}$ :

$$
\begin{aligned}
\sigma_{q_{i} \bar{q}_{j} \rightarrow \tilde{q} \overline{\tilde{q}}, \mathbf{1}}^{(0)} & =\frac{8}{9} \frac{\pi \hat{\alpha}_{\mathrm{s}}^{2}}{\hat{s}}\left[\beta_{\tilde{q}}\left(-\frac{4}{9}-\frac{4 m_{-}^{4}}{9\left(m_{\tilde{g}}^{2} \hat{s}+m_{-}^{4}\right)}\right)-\left(\frac{4}{9}+\frac{8 m_{-}^{2}}{9 \hat{s}}\right) L_{1}\right], \\
\sigma_{q_{i} \bar{q}_{j} \rightarrow \tilde{q} \overline{\tilde{q}}, \mathbf{8}}^{(0)} & =\delta_{i j} \frac{n_{\mathrm{f}} \pi \alpha_{\mathrm{s}}^{2}}{\hat{s}} \frac{4}{27} \beta_{\tilde{q}}^{3} \\
& +\delta_{i j} \frac{\pi \alpha_{\mathrm{s}} \hat{\alpha}_{\mathrm{s}}}{\hat{s}}\left[\beta_{\tilde{q}}\left(\frac{4}{27}+\frac{8 m_{-}^{2}}{27 \hat{s}}\right)+\left(\frac{8 m_{\tilde{g}}^{2}}{27 \hat{s}}+\frac{8 m_{-}^{4}}{27 \hat{s}^{2}}\right) L_{1}\right] \\
& +\frac{1}{9} \frac{\pi \hat{\alpha}_{\mathrm{s}}^{2}}{\hat{s}}\left[\beta_{\tilde{q}}\left(-\frac{4}{9}-\frac{4 m_{-}^{4}}{9\left(m_{\tilde{g}}^{2} \hat{s}+m_{-}^{4}\right)}\right)-\left(\frac{4}{9}+\frac{8 m_{-}^{2}}{9 \hat{s}}\right) L_{1}\right],
\end{aligned}
$$

for $g g \rightarrow \tilde{q} \overline{\tilde{q}}$ :

$$
\sigma_{g g \rightarrow \tilde{q} \overline{\tilde{q}}, \mathbf{1}}^{(0)}=\frac{n_{\mathrm{f}} \pi \alpha_{\mathrm{s}}^{2}}{\hat{s}}\left[\beta_{\tilde{q}}\left(\frac{1}{24}+\frac{m_{\tilde{q}}^{2}}{6 \hat{s}}\right)+\left(\frac{m_{\tilde{q}}^{2}}{6 \hat{s}}-\frac{m_{\tilde{q}}^{4}}{3 \hat{s}^{2}}\right) \log \left(\frac{1-\beta_{\tilde{q}}}{1+\beta_{\tilde{q}}}\right)\right]
$$




$$
\sigma_{g g \rightarrow \tilde{q} \tilde{\tilde{q}}, \mathbf{8}_{\mathbf{S}}}^{(0)}+\sigma_{g g \rightarrow \tilde{q} \tilde{\tilde{q}}, \mathbf{8}_{\mathbf{A}}}^{(0)}=\frac{n_{\mathrm{f}} \pi \alpha_{\mathbf{S}}^{2}}{\hat{s}}\left[\beta_{\tilde{q}}\left(\frac{1}{6}+\frac{29 m_{\tilde{q}}^{2}}{12 \hat{s}}\right)+\left(\frac{7 m_{\tilde{q}}^{2}}{6 \hat{s}}+\frac{2 m_{\tilde{q}}^{4}}{3 \hat{s}^{2}}\right) \log \left(\frac{1-\beta_{\tilde{q}}}{1+\beta_{\tilde{q}}}\right)\right],
$$

for $q \bar{q} \rightarrow \tilde{g} \tilde{g}:$

$$
\begin{aligned}
& \sigma_{q \bar{q} \rightarrow \tilde{g} \tilde{g}, \mathbf{1}}^{(0)}=\frac{\pi \hat{\alpha}_{\mathbf{s}}^{2}}{\hat{s}}\left[\beta_{\tilde{g}}\left(\frac{4}{27}+\frac{4 m_{-}^{4}}{27\left(m_{\tilde{q}}^{2} \hat{s}+m_{-}^{4}\right)}\right)-\left(\frac{8 m_{-}^{2}}{27 \hat{s}}-\frac{8 m_{\tilde{g}}^{2}}{27\left(\hat{s}-2 m_{-}^{2}\right)}\right) L_{2}\right], \\
& \sigma_{q \bar{q} \rightarrow \tilde{g} \tilde{g}, \mathbf{8}_{\mathbf{S}}}^{(0)}+\sigma_{q \bar{q} \rightarrow \tilde{g} \tilde{g}, \mathbf{8}_{\mathbf{A}}}^{(0)}=\frac{\pi \alpha_{\mathbf{S}}^{2}}{\hat{s}} \beta_{\tilde{g}}\left(\frac{8}{9}+\frac{16 m_{\tilde{g}}^{2}}{9 \hat{s}}\right) \\
+ & \frac{\pi \alpha_{\mathrm{s}} \hat{\alpha}_{\mathbf{s}}}{\hat{s}}\left[\beta_{\tilde{g}}\left(-\frac{4}{3}-\frac{8 m_{-}^{2}}{3 \hat{s}}\right)+\left(\frac{8 m_{\tilde{g}}^{2}}{3 \hat{s}}+\frac{8 m_{-}^{4}}{3 \hat{s}^{2}}\right) L_{2}\right] \\
+ & \frac{\pi \hat{\alpha}_{\mathbf{s}}^{2}}{\hat{s}}\left[\beta_{\tilde{g}}\left(\frac{28}{27}+\frac{28 m_{-}^{4}}{27\left(m_{\tilde{q}}^{2} \hat{s}+m_{-}^{4}\right)}\right)-\left(\frac{56 m_{-}^{2}}{27 \hat{s}}+\frac{16 m_{\tilde{g}}^{2}}{27\left(\hat{s}-2 m_{-}^{2}\right)}\right) L_{2}\right],
\end{aligned}
$$

and for $g g \rightarrow \tilde{g} \tilde{g}$ :

$$
\begin{aligned}
\sigma_{g g \rightarrow \tilde{g} \tilde{g}, \mathbf{1}}^{(0)} & =\frac{1}{2} \sigma_{\mathrm{sym}}, \\
\sigma_{g g \rightarrow \tilde{g} \tilde{g}, \mathbf{8}_{\mathbf{S}}}^{(0)}+\sigma_{g g \rightarrow \tilde{g} \tilde{g}, \mathbf{8}_{\mathbf{A}}}^{(0)} & =\sigma_{\mathrm{sym}}+\sigma_{\mathrm{asym}}, \\
\sigma_{g g \rightarrow \tilde{g} \tilde{g}, \mathbf{1 0}}^{(0)} & =0, \\
\sigma_{g g \rightarrow \tilde{g} \tilde{g}, \mathbf{2 7}}^{(0)} & =\frac{3}{2} \sigma_{\mathrm{sym}}, \\
\sigma_{g g \rightarrow \tilde{g} \tilde{g}, I}^{(0)} & =0 \text { for } \quad I=6 \ldots 8,
\end{aligned}
$$

with

$$
\begin{aligned}
\sigma_{\text {asym }}= & \frac{\pi \alpha_{\mathrm{s}}^{2}}{\hat{s}}\left[\beta_{\tilde{g}}\left(-\frac{21}{16}-\frac{6 m_{\tilde{g}}^{2}}{\hat{s}}\right)\right. \\
& \left.-\left(\frac{9}{16}+\frac{9 m_{\tilde{g}}^{2}}{4 \hat{s}}+\frac{9 m_{\tilde{g}}^{4}}{2 \hat{s}^{2}}\right) \log \left(\frac{1-\beta_{\tilde{g}}}{1+\beta_{\tilde{g}}}\right)\right], \\
\sigma_{\mathrm{sym}}= & \frac{\pi \alpha_{\mathrm{s}}^{2}}{\hat{s}}\left[\beta_{\tilde{g}}\left(-\frac{9}{16}-\frac{9 m_{\tilde{g}}^{2}}{4 \hat{s}}\right)\right. \\
& \left.-\left(\frac{9}{16}+\frac{9 m_{\tilde{g}}^{2}}{4 \hat{s}}-\frac{9 m_{\tilde{g}}^{4}}{2 \hat{s}^{2}}\right) \log \left(\frac{1-\beta_{\tilde{g}}}{1+\beta_{\tilde{g}}}\right)\right],
\end{aligned}
$$

and

$$
\begin{gathered}
\beta_{\tilde{q}}=\sqrt{1-\frac{4 m_{\tilde{q}}^{2}}{\hat{s}}}, \quad \beta_{\tilde{g}}=\sqrt{1-\frac{4 m_{\tilde{g}}^{2}}{\hat{s}}}, \quad m_{-}^{2}=m_{\tilde{g}}^{2}-m_{\tilde{q}}^{2}, \\
L_{1}=\log \left(\frac{\hat{s}\left(1-\beta_{\tilde{q}}\right)+2 m_{-}^{2}}{\hat{s}\left(1+\beta_{\tilde{q}}\right)+2 m_{-}^{2}}\right), \quad L_{2}=\log \left(\frac{\hat{s}\left(1-\beta_{\tilde{g}}\right)-2 m_{-}^{2}}{\hat{s}\left(1+\beta_{\tilde{g}}\right)-2 m_{-}^{2}}\right),
\end{gathered}
$$

where $\hat{\alpha}_{\mathrm{s}}$ is the SUSY Yukawa coupling.

\section{Threshold resummation - general framework}

The resummation for $2 \rightarrow 2$ processes with all four external legs carrying colour was studied extensively in the literature. The resummed cross section for the heavy-quark production was 
constructed in [19, 23], and for the dijet (multiple jet) production in [20, 21, 22]. Here we briefly review the derivation of the resummed cross sections for the production of two coloured final state particles of equal mass $m$, in the form presented in [23]. In our calculations me make use of the framework of $[19,20,21]$.

Using the hadronic threshold variable $\rho \equiv 4 \mathrm{~m}^{2} / S$ we rewrite the cross section (1)

$$
\sigma_{h_{1} h_{2} \rightarrow k l}\left(\rho,\left\{m^{2}\right\}\right)=\sum_{i, j} \sum_{I} \int d x_{1} d x_{2} d \hat{\rho} \delta\left(\hat{\rho}-\frac{\rho}{x_{1} x_{2}}\right) f_{i / h_{1}}\left(x_{1}, \mu\right) f_{j / h_{2}}\left(x_{2}, \mu\right) \sigma_{i j \rightarrow k l, I}\left(\hat{\rho},\left\{m^{2}\right\}, \mu^{2}\right),
$$

where the index $I$ sums over all possible colour states of the hard scattering.

At higher orders in perturbation theory, the partonic cross section $\hat{\sigma}$ contains terms of general structure $\alpha_{\mathrm{s}}^{n} \log ^{m} \beta^{2}, m \leq 2 n$, with $\beta=\sqrt{1-\hat{\rho}}$. These terms are singular in the threshold limit $\hat{\rho} \rightarrow 1$. The singularities can be systematically treated by taking Mellin moments of the cross section

$$
\begin{aligned}
\tilde{\sigma}_{h_{1} h_{2} \rightarrow k l}\left(N,\left\{m^{2}\right\}\right) & \equiv \int_{0}^{1} d \rho \rho^{N-1} \sigma_{h_{1} h_{2} \rightarrow k l}\left(\rho,\left\{m^{2}\right\}\right) \\
& =\sum_{i, j} \tilde{f}_{i / h_{1}}\left(N+1, \mu^{2}\right) \tilde{f}_{j / h_{2}}\left(N+1, \mu^{2}\right) \tilde{\sigma}_{i j \rightarrow k l}\left(N,\left\{m^{2}\right\}, \mu^{2}\right) .
\end{aligned}
$$

The moments of the parton distributions $f_{i / h}\left(x_{i}, \mu^{2}\right)$ are defined in the standard way, ${ }^{1}$

$$
\tilde{f}_{i / h}\left(N, \mu^{2}\right) \equiv \int_{0}^{1} d x x^{N-1} f_{i / h}\left(x, \mu^{2}\right),
$$

and the moments of the partonic cross section $a b \rightarrow k l$ are given by

$$
\tilde{\sigma}_{i j \rightarrow k l}\left(N,\left\{m^{2}\right\}, \mu^{2}\right) \equiv \int_{0}^{1} d \hat{\rho} \hat{\rho}^{N-1} \sigma_{i j \rightarrow k l}\left(\hat{\rho},\left\{m^{2}\right\}, \mu^{2}\right) .
$$

Taking the Mellin moments transforms the logarithms in $\beta^{2}$ into the logarithms of the Mellin variable $N$ which are then resummed to all orders in $\alpha_{\mathrm{s}}$.

Following [27] we define the differential distribution

$$
\begin{aligned}
& \frac{d \sigma_{h_{1} h_{2} \rightarrow k l}}{d \xi}\left(\rho,\left\{m^{2}\right\}\right)= \\
= & \sum_{i, j} \sum_{I} \int d x_{1} d x_{2} f_{i / h_{1}}\left(x_{1}, \mu\right) f_{j / h_{2}}\left(x_{2}, \mu\right) \delta\left(\xi-x_{1} x_{2}\right) \sigma_{i j \rightarrow k l, I}\left(\rho / \xi,\left\{m^{2}\right\}, \mu^{2}\right) \\
= & \sum_{i, j} \sum_{I} \sigma_{i j \rightarrow k l, I}\left(\rho / \xi,\left\{m^{2}\right\}, \mu^{2}\right) \int_{C-i \infty}^{C+i \infty} d N \xi^{-N} \tilde{f}_{i / h_{1}}\left(N, \mu^{2}\right) \tilde{f}_{j / h_{2}}\left(N, \mu^{2}\right) .
\end{aligned}
$$

Since soft radiation carries colour charge, it can change the colour state of the underlying hard scattering for hadronic processes with two or more coloured partons in the final state. This

\footnotetext{
${ }^{1}$ Note that from now on we will use the tilde sign to mark symbols for $N$-space quantities.
} 
has to be taken into account while writing the form of the cross section with long-distance and short-distance effects factorised, and leads to [20, 23]

$$
\begin{aligned}
\frac{d \sigma_{h_{1} h_{2} \rightarrow k l}^{(\mathrm{res})}}{d \xi}\left(\rho,\left\{m^{2}\right\}\right) & =\sum_{i, j} \sum_{I, J} h_{i j \rightarrow k l, I}^{*}\left(\rho / \xi,\left\{m^{2}\right\}, \mu^{2}\right) h_{i j \rightarrow k l, J}\left(\rho / \xi,\left\{m^{2}\right\}, \mu^{2}\right) \\
& \times \frac{1}{2 \pi i} \int_{C-i \infty}^{C+i \infty} d N \xi^{-N} \tilde{f}_{i / h_{1}}\left(N, \mu^{2}\right) \tilde{f}_{j / h_{2}}\left(N, \mu^{2}\right) \tilde{\omega}_{i j \rightarrow k l, I J}^{(\mathrm{res})}(N, Q, \mu),
\end{aligned}
$$

with $Q^{2}=4 m^{2}$. The function $h_{i j \rightarrow k l, I}\left(h_{i j \rightarrow k l, J}^{*}\right)$ is a colour-dependent hard-scattering amplitude (conjugate of) absorbing the far, i.e. of the order of the scale of the process $Q$, off-shell effects. All the logarithmic dependence on $N$, originating from soft and collinear radiation, is contained in the function $\tilde{\omega}_{i j \rightarrow k l, I J}^{(\text {res })}(N, Q, \mu)$.

In the approach of $[19,20,21,28]$, resummation follows from refactorisation of partonic cross sections. In the case of threshold resummation, the cross sections are factorised w.r.t. fixed fractions of energy as opposed to fractions of momenta in the standard collinear factorisation. Using the refactorised form of the cross section for the production of two massive coloured particles in the final state [19], we have, up to corrections of $\mathcal{O}(1 / N)$,

$$
\begin{aligned}
\frac{d \sigma_{h_{1} h_{2} \rightarrow k l}^{(\mathrm{res})}}{d \xi}\left(\rho,\left\{m^{2}\right\}\right) & =\sum_{i, j} \sum_{I, J} h_{i j \rightarrow k l, I}^{*}\left(\rho / \xi,\left\{m^{2}\right\}, \mu^{2}\right) h_{i j \rightarrow k l, J}\left(\rho / \xi,\left\{m^{2}\right\}, \mu^{2}\right) \\
& \times \frac{1}{2 \pi i} \int_{C-i \infty}^{C+i \infty} d N \xi^{-N} \tilde{f}_{i / h_{1}}\left(N, \mu^{2}\right) \tilde{f}_{j / h_{2}}\left(N, \mu^{2}\right) \\
& \times \frac{\tilde{\psi}_{i / i}\left(N, Q / \mu, \alpha_{\mathrm{s}}\left(\mu^{2}\right)\right) \tilde{\psi}_{j / j}\left(N, Q / \mu, \alpha_{\mathrm{s}}\left(\mu^{2}\right)\right)}{\tilde{f}_{i / i}\left(N, \mu^{2}\right) \tilde{f}_{j / j}\left(N, \mu^{2}\right)} \tilde{S}_{i j \rightarrow k l, I J}\left(Q /(N \mu), \alpha_{\mathrm{s}}\left(\mu^{2}\right)\right) .
\end{aligned}
$$

Following $[19,20]$ the soft eikonal function $\tilde{S}$ represents coupling of the soft gluons to the initial and final state particles. Consequently, the soft function carries dependence on the possible colour exchanges and we sum over all possible colour structures $I, J$ at hard vertices. As an object of purely eikonal character $[19,28,29]$ the soft function can depend on the scales only through their ratio. The parton-in-parton distributions $\psi_{i / i}$ are defined at fixed fraction of energy of parton $i$ in the partonic center-of-mass frame as opposed to the light-cone distributions $f_{i / i}$ which are defined at fixed momentum fraction. The parton distribution functions and the soft function can be defined explicitly in terms of operator matrix elements [20].

As a consequence of refactorisation, the soft function $\tilde{S}_{i j, I J}$ and the distributions functions $\tilde{\psi}_{i / i}, \tilde{f}_{i / i}$ obey the corresponding renormalisation group equations (RGEs) [19, 20, 21, 28]. Solutions of these RGEs give functions which resum the large logarithms in question. In fact for the $2 \rightarrow 2$ production process involving coloured massive particles only the solution of the RGE for the soft function

$$
\left(\mu \frac{\partial}{\partial \mu}+\beta(g) \frac{\partial}{\partial g}\right) \tilde{S}_{i j \rightarrow k l, I J}=-\Gamma_{i j \rightarrow k l, I K}^{\dagger} \tilde{S}_{i j \rightarrow k l, K J}-\tilde{S}_{i j \rightarrow k l, I L} \Gamma_{i j \rightarrow k l, L J}
$$


is needed. As shown in [19], the resummed initial-state jet factors for the ratio of $\tilde{\psi}_{i / i}$ to $\tilde{f}_{i / i}$ functions in Eq. (28) can be obtained directly from the resummed cross section for production of a colour singlet state through the Drell-Yan mechanism, for which results are known [28, 30]. We write this ratio in the form

$$
\frac{\tilde{\psi}_{i / i}\left(N, Q / \mu, \alpha_{\mathrm{s}}\left(\mu^{2}\right)\right)}{\tilde{f}_{i / i}\left(N, \mu^{2}\right)}=R_{i}\left(\alpha_{\mathrm{s}}\left(\mu^{2}\right)\right) \Delta_{i}\left(N, Q^{2}, \mu^{2}\right)\left[\tilde{U}_{i \bar{i}}\left(Q /(N \mu), \alpha_{\mathrm{s}}\left(\mu^{2}\right)\right)\right]^{-1 / 2}
$$

where the function $R_{i}\left(\alpha_{\mathrm{s}}\left(\mu^{2}\right)\right)$ is an $N$-independent and infrared-safe function of the coupling. The soft eikonal function $\tilde{U}_{i \bar{i}}$ describes soft gluon emission and exchange by the annihilating initial state partons in the Drell-Yan process. The radiative factor $\Delta_{i}$ represents both the soft and collinear radiation from an incoming parton. Inserting the expression for the ratio of $\psi$ to $f$ functions, Eq. (30), into Eq. (28) leads to

$$
\begin{aligned}
\frac{d \sigma_{h_{1} h_{2} \rightarrow k l}^{(\mathrm{res})}}{d \xi}\left(\rho,\left\{m^{2}\right\}\right) & =\sum_{i, j} \sum_{I, J} h_{i j \rightarrow k l, I}^{*}\left(\rho / \xi,\left\{m^{2}\right\}, \mu^{2}\right) h_{i j \rightarrow k l, J}\left(\rho / \xi,\left\{m^{2}\right\}, \mu^{2}\right) \\
& \times \frac{1}{2 \pi i} \int_{C-i \infty}^{C+i \infty} d N \xi^{-N} \tilde{f}_{i / h_{1}}\left(N, \mu^{2}\right) \tilde{f}_{j / h_{2}}\left(N, \mu^{2}\right) R_{i}\left(\alpha_{\mathrm{s}}\left(\mu^{2}\right)\right) R_{j}\left(\alpha_{\mathrm{s}}\left(\mu^{2}\right)\right) \\
& \times \Delta_{i}\left(N, Q^{2}, \mu^{2}\right) \Delta_{j}\left(N, Q^{2}, \mu^{2}\right) \overline{\tilde{S}}_{i j \rightarrow k l, I J}\left(Q /(N \mu), \alpha_{\mathrm{s}}\left(\mu^{2}\right)\right)
\end{aligned}
$$

where we introduce $\overline{\tilde{S}}_{i j \rightarrow k l, I J} \equiv \tilde{S}_{i j \rightarrow k l, I J} / \tilde{U}_{i \bar{i}}$. Consequently, the soft anomalous dimension matrix $\bar{\Gamma}_{i j \rightarrow k l}$ corresponding to the function $\overline{\tilde{S}}_{i j \rightarrow k l, I J}$ is given by

$$
\bar{\Gamma}_{i j \rightarrow k l, I J}\left(\alpha_{\mathrm{s}}\right)=\Gamma_{i j \rightarrow k l, I J}\left(\alpha_{\mathrm{s}}\right)-\delta_{I J} \Gamma_{i \bar{i}}\left(\alpha_{\mathrm{s}}\right)
$$

where $\Gamma_{i \bar{i}}$ is the anomalous dimension associated with the Drell-Yan soft function $\tilde{U}_{i \bar{i}}$. The results for $\Gamma_{i \bar{i}}$ can be found in the literature $[21,28]$.

In general, for a given colour basis, the soft anomalous dimension matrix $\Gamma_{I J}$ is not diagonal, leading to resummed expressions in terms of path-ordered exponentials [20]. Through the diagonalisation of the soft anomalous dimension matrix a simpler form for the resummed cross sections, involving a sum of exponentials, can be obtained. However, the diagonalisation procedure can be avoided if from the beginning the calculations are performed in the colour basis in which the the soft anomalous dimension matrix is diagonal, i.e.

$$
\Gamma_{i j \rightarrow k l}\left(\alpha_{\mathrm{s}}\right)=\operatorname{diag}\left(\ldots, \lambda_{i j \rightarrow k l, I}\left(\alpha_{\mathrm{s}}\right), \ldots\right)
$$

The soft function then reads, up to NLL,

$$
\begin{aligned}
\tilde{S}_{i j \rightarrow k l, I J}\left(Q /(N \mu), \alpha_{\mathrm{S}}\left(\mu^{2}\right)\right) & =\tilde{S}_{i j \rightarrow k l, I J}^{(0)} \\
& \times \exp \left[\int_{\mu}^{Q / N} \frac{d q}{q}\left[\lambda_{i j \rightarrow k l, I}^{*}\left(\alpha_{\mathrm{S}}\left(q^{2}\right)\right)+\lambda_{i j \rightarrow k l, J}\left(\alpha_{\mathrm{S}}\left(q^{2}\right)\right)\right]\right],
\end{aligned}
$$


with the 0 -th order term in the perturbative expansion of $\tilde{S}_{i j \rightarrow k l, I J}\left(1, \alpha_{\mathrm{S}}\left(Q^{2} / N^{2}\right)\right)$ straightforwardly related to the colour structure, i.e.

$$
\tilde{S}_{i j \rightarrow k l, I J}^{(0)}=\operatorname{Tr}\left(c_{I}^{\dagger} c_{J}\right)
$$

where $\left\{c_{I}\right\}$ is the corresponding colour basis for the process $i j \rightarrow k l$, see Section 2 . If additionally the colour basis is orthogonal then the soft function matrix becomes diagonal

$$
\tilde{S}_{i j \rightarrow k l, I J}\left(Q /(N \mu), \alpha_{\mathrm{s}}\left(\mu^{2}\right)\right)=\delta_{I J} \tilde{S}_{i j \rightarrow k l, I I}^{(0)} \exp \left[\int_{\mu}^{Q / N} \frac{d q}{q} 2 \operatorname{Re}\left(\lambda_{i j \rightarrow k l, I}\left(\alpha_{\mathrm{s}}\left(q^{2}\right)\right)\right)\right] .
$$

In this case inserting the solution (36) into Eq. (31) leads to the following NLL expression

$$
\begin{aligned}
\frac{d \sigma_{h_{1} h_{2} \rightarrow k l}^{(\mathrm{res})}}{d \xi}\left(\rho,\left\{m^{2}\right\}\right) & =\sum_{i, j} \sum_{I} \frac{1}{2 \pi i} \int_{C-i \infty}^{C+i \infty} d N \xi^{-N} \\
& \times \hat{\sigma}_{i j \rightarrow k l, I}^{(0)}\left(\rho / \xi,\left\{m^{2}\right\}, \mu^{2}\right) \mathcal{C}_{i j \rightarrow k l, I}\left(\rho / \xi, N,\left\{m^{2}\right\}, \mu^{2}\right) \\
& \times \tilde{f}_{i / h_{1}}\left(N, \mu^{2}\right) \tilde{f}_{j / h_{2}}\left(N, \mu^{2}\right) \Delta_{i}\left(N, Q^{2}, \mu^{2}\right) \Delta_{j}\left(N, Q^{2}, \mu^{2}\right) \Delta_{i j \rightarrow k l, I}^{(s)}\left(N, Q^{2}, \mu^{2}\right),
\end{aligned}
$$

where we identify

$$
\left|h_{i j \rightarrow k l, I}\right|^{2} R_{i} R_{j} \tilde{S}_{i j \rightarrow k l, I I}^{(0)} \equiv \sigma_{i j \rightarrow k l, I}^{(0)} \mathcal{C}_{i j \rightarrow k l, I} .
$$

The functions $\mathcal{C}_{i j \rightarrow k l, I}$ are of perturbative nature and contain information about higher-order corrections which are non-logarithmic in $N$. All the information on the soft non-collinear logarithmic corrections is included in the radiative factor $\Delta_{i j \rightarrow k l, I}^{(s)}$. After performing integration over $\xi$ we obtain

$$
\begin{aligned}
\sigma_{h_{1} h_{2} \rightarrow k l}^{(\mathrm{res})}\left(\rho,\left\{m^{2}\right\}\right) & =\sum_{i, j} \sum_{I} \frac{1}{2 \pi i} \int_{C-i \infty}^{C+i \infty} d N \rho^{-N+1} \tilde{f}_{i / h_{1}}\left(N, \mu^{2}\right) \tilde{f}_{j / h_{2}}\left(N, \mu^{2}\right) \\
& \times \tilde{\sigma}_{i j \rightarrow k l, I}^{(0)}\left(N-1,\left\{m^{2}\right\}, \mu^{2}\right) \overline{\mathcal{C}}_{i j \rightarrow k l, I}\left(N-1,\left\{m^{2}\right\}, \mu^{2}\right) \\
& \times \Delta_{i}\left(N, Q^{2}, \mu^{2}\right) \Delta_{j}\left(N, Q^{2}, \mu^{2}\right) \Delta_{i j \rightarrow k l, I}^{(s)}\left(N, Q^{2}, \mu^{2}\right) .
\end{aligned}
$$

The functions $\overline{\mathcal{C}}_{i j \rightarrow k l, I}$ are related to the $\mathcal{C}_{i j \rightarrow k l, I}$ functions in Eq. (37) and have a perturbative expansion of the form $\overline{\mathcal{C}}_{i j \rightarrow k l, I}=1+\sum_{n=1} \alpha_{\mathrm{s}}^{n} \overline{\mathcal{C}}_{i j \rightarrow k l, I}^{(n)}$. In general, the values of the coefficients $\overline{\mathcal{C}}_{i j \rightarrow k l, I}^{(n)}$ are obtained by comparing the resummed cross section expanded in $\alpha_{\mathrm{s}}$ with the expression for the full higher-order cross section in $N$ space. The expression for the resummed hadronic cross section in the Mellin-moment space can be easily derived from Eq. (39) and reads

$$
\begin{aligned}
& \sigma_{h_{1} h_{2} \rightarrow k l}^{(\mathrm{res})}\left(N,\left\{m^{2}\right\}\right)=\sum_{i, j} \sum_{I} \tilde{f}_{i / h_{1}}\left(N+1, \mu^{2}\right) \tilde{f}_{j / h_{2}}\left(N+1, \mu^{2}\right) \tilde{\sigma}_{i j \rightarrow k l, I}^{(0)}\left(N,\left\{m^{2}\right\}, \mu^{2}\right) \\
& \times \overline{\mathcal{C}}_{i j \rightarrow k l, I}\left(N,\left\{m^{2}\right\}, \mu^{2}\right) \Delta_{i}\left(N+1, Q^{2}, \mu^{2}\right) \Delta_{j}\left(N+1, Q^{2}, \mu^{2}\right) \Delta_{i j \rightarrow k l, I}^{(s)}\left(N+1, Q^{2}, \mu^{2}\right) .
\end{aligned}
$$

The expressions for the radiative factors $\Delta_{i}, \Delta_{i j \rightarrow k l, I}^{(s)}$ are presented below. Since after expansion of the exponentials the non-trivial terms contained in $\overline{\mathcal{C}}_{i j \rightarrow k l, I}$ generate contributions of the 
NNLL and higher orders, we keep $\overline{\mathcal{C}}_{i j \rightarrow k l, I}=1$ for the rest of the calculations. Additionally, in Appendix A we list the results for the Mellin moments of the colour-channel contributions $\tilde{\sigma}_{i j \rightarrow k l, I}^{(0)}$ to the leading-order partonic cross sections.

\subsection{Soft radiation factors}

The expressions for the radiative factors in the $\overline{\mathrm{MS}}$ factorisation scheme read, up to the NLL level [19, 20, 23],

$$
\begin{aligned}
& \log \Delta_{i}\left(N, Q^{2}, \mu^{2}\right)=\int_{0}^{1} d z \frac{z^{N-1}-1}{1-z} \int_{\mu^{2}}^{Q^{2}(1-z)^{2}} \frac{d q^{2}}{q^{2}} A_{i}\left(\alpha_{\mathrm{S}}\left(q^{2}\right)\right), \\
& \log \Delta_{i j \rightarrow k l, I}^{(s)}\left(N, Q^{2}, \mu^{2}\right)=\int_{0}^{1} d z \frac{z^{N-1}-1}{1-z} \frac{\alpha_{\mathrm{s}}\left((1-z)^{2} Q^{2}\right)}{\pi} D_{i j \rightarrow k l, I}^{(1)} .
\end{aligned}
$$

As already noted, the radiative factor $\Delta_{i}$ represents the soft and collinear gluon radiation from the incoming partons, whereas the function $\Delta_{i j \rightarrow k l, I}^{(s)}$ takes into account soft and large-angle gluon radiation. The coefficient $A_{i}$ is a power series in the coupling constant $\alpha_{\mathrm{s}}, A_{i}\left(\alpha_{\mathrm{s}}\right)=$ $\frac{\alpha_{\mathrm{s}}}{\pi} A_{i}{ }^{(1)}+\left(\frac{\alpha_{\mathrm{s}}}{\pi}\right)^{2} A_{i}^{\left({ }^{(2)}\right.}+\ldots$ The universal leading logarithm (LL) and NLL coefficients $A_{i}^{(1)}, A_{i}^{(2)}$ are well known $[31,32]$ and given by

$$
A_{i}^{(1)}=C_{i}, \quad A_{i}^{(2)}=\frac{1}{2} C_{i}\left(\left(\frac{67}{18}-\frac{\pi^{2}}{6}\right) C_{\mathrm{A}}-\frac{5}{9} n_{\mathrm{f}}\right),
$$

with $C_{g}=C_{\mathrm{A}}=3$ for radiation off gluon lines and $C_{q}=C_{\mathrm{F}}=4 / 3$ for radiation off quark lines. In the case the soft anomalous dimension matrix $\Gamma_{i j \rightarrow k l}$ is diagonal in the orthogonal colour basis, the relation between the $D_{i j \rightarrow k l, I}^{(1)}$ coefficients and the $\bar{\Gamma}_{i j \rightarrow k l}$ eigenvalues, $\bar{\lambda}_{i j \rightarrow k l, I}$, reads $[19,20$, $21]$

$$
\frac{\alpha_{\mathrm{s}}}{\pi} D_{i j \rightarrow k l, I}^{(1)}=2 \operatorname{Re}\left(\bar{\lambda}_{i j \rightarrow k l, I}\left(\alpha_{\mathrm{s}}\right)\right) .
$$

The customary NLL expansions of the radiative factors (41) are presented in Appendix B.

\section{Soft anomalous dimension matrices}

Due to the same colour structure the soft anomalous dimension matrices for the $\tilde{q} \overline{\tilde{q}}$ production and for the heavy quark production are the same. The results for the heavy quark production are available in the literature [19]. For the $\tilde{g} \tilde{g}$ production, however, a separate calculation is needed.

In order to obtain resummed cross sections up to the NLL accuracy we need the one-loop $\mathcal{O}\left(\alpha_{\mathrm{s}}\right)$ result for the soft anomalous dimension matrix, $\Gamma_{i j \rightarrow k l, I J}^{(1)}$. The $\Gamma_{i j \rightarrow k l, I J}$ matrices are given in terms of matrices of renormalisation constants $Z_{i j \rightarrow k l, I J}$ for the soft function [18]-[21], [29]. In the $\overline{\mathrm{MS}}$ scheme in the $d=4-\epsilon$ dimensions we have

$$
\Gamma_{i j \rightarrow k l, I J}^{(1)}\left(g_{s}\right)=-\frac{g_{s}}{2} \frac{\partial}{\partial g_{s}} \operatorname{Res}_{\epsilon \rightarrow 0} Z_{i j \rightarrow k l, I J}^{(1)}\left(g_{s}, \epsilon\right) .
$$



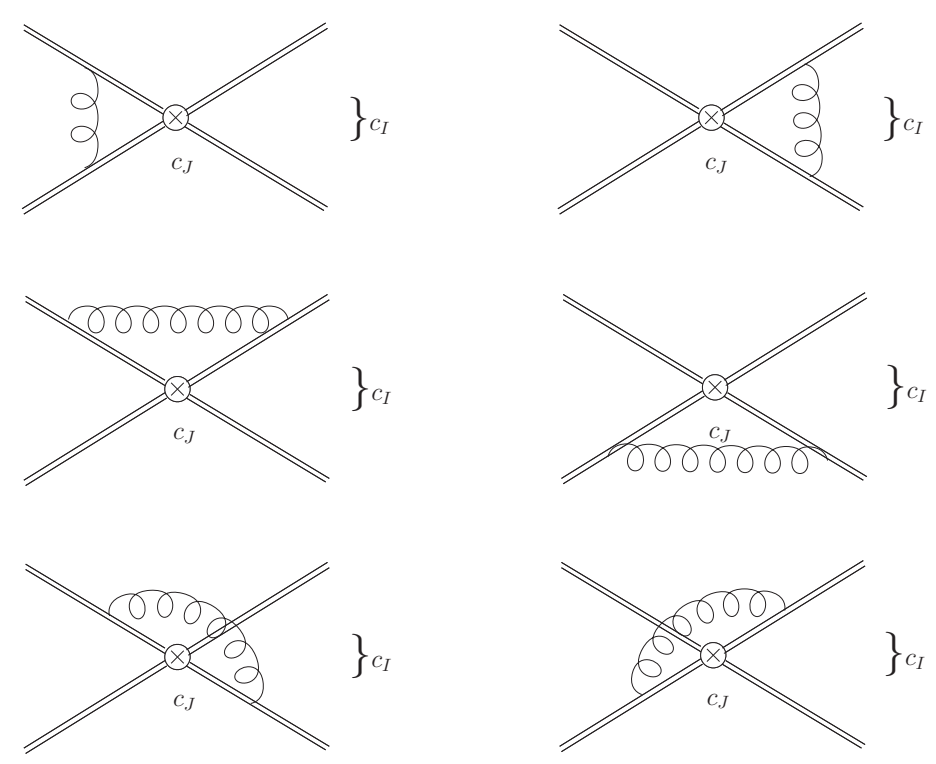

(a)
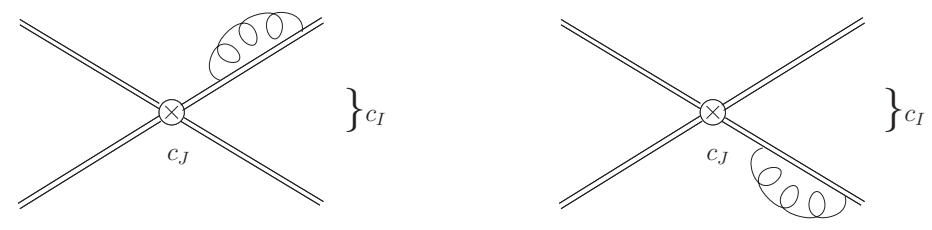

(b)

Figure 1: Vertex (a) and self-energy (b) one-loop contributions to the soft function $S_{i j \rightarrow k l, I J}$ with $i, j$ massless and $k, l$ massive particles.

The calculation of $\Gamma_{i j \rightarrow k l, I J}^{(1)}$ reduces then to evaluating the UV-divergent part of the one-loop correction to the $S_{i j \rightarrow k l, I J}$ function.

In Fig. 1, following [19], we show the set of eikonal one-loop diagrams contributing to the soft function $S_{i j \rightarrow k l, I J}$. All one-loop integrals needed for the calculation of the anomalous dimension matrices for $q \bar{q} \rightarrow \tilde{g} \tilde{g}$ and $g g \rightarrow \tilde{g} \tilde{g}$ are known. In our calculation we use the results for the eikonal one-loop integrals from Ref. $[18,19]$ which have been obtained in the axial gauge. Although the integrals are the same as in the heavy-quark production case, their contributions to the soft anomalous dimension matrices come weighted with different colour factors. We compute these colour factors in the set of basis presented in Section 2. The calculations are performed in two independent ways: using the FeynCalc package [33] for Mathematica, and using the Group Theory (Colour) Factors of Feynman diagrams package [34] for FORM [35]. The resulting expressions for the soft anomalous dimension matrices for the $\tilde{g} \tilde{g}$ production are presented below. 


\subsection{Gluino-pair production}

We introduce the notation

$$
\begin{aligned}
\bar{T} & \equiv \log \left(\frac{m^{2}-\hat{t}}{\sqrt{m^{2} \hat{s}}}\right)-\frac{1-i \pi}{2} \\
\bar{U} & \equiv \log \left(\frac{m^{2}-\hat{u}}{\sqrt{m^{2} \hat{s}}}\right)-\frac{1-i \pi}{2} \\
\bar{S} & \equiv-\frac{L_{\beta}+1}{2}
\end{aligned}
$$

where the Mandelstam variables are given by

$$
\hat{s}=\left(p_{1}+p_{2}\right)^{2}, \quad \hat{t}=\left(p_{1}-p_{3}\right)^{2}, \quad \hat{u}=\left(p_{1}-p_{4}\right)^{2},
$$

and $L_{\beta}=\frac{1}{\beta}\left(1-2 m^{2} / \hat{s}\right)\left(\log \frac{1-\beta}{1+\beta}+i \pi\right)$. We also define $\Lambda \equiv \bar{T}+\bar{U}, \Omega \equiv \bar{T}-\bar{U}$.

In the basis (5) we obtain the one-loop soft anomalous dimension matrix of the form

$$
\bar{\Gamma}_{q \bar{q} \rightarrow \tilde{g} \tilde{g}}^{(1)}\left(\alpha_{\mathrm{S}}\right)=\frac{\alpha_{\mathrm{s}}}{\pi}\left[\left(\begin{array}{ccc}
6 \bar{S} & 0 & -\Omega \\
0 & 3 \bar{S}+\frac{3}{2} \Lambda & -\frac{3}{2} \Omega \\
-2 \Omega & -\frac{5}{6} \Omega & 3 \bar{S}+\frac{3}{2} \Lambda
\end{array}\right)-\frac{4}{3} i \pi \hat{\mathbf{I}}\right] .
$$

The one-loop soft anomalous dimension matrix for the $g g$ channel, calculated in the basis (8) has the block form

$$
\bar{\Gamma}_{g g \rightarrow \tilde{g} \tilde{g}}^{(1)}\left(\alpha_{\mathrm{S}}\right)=\frac{\alpha_{\mathrm{s}}}{\pi}\left[\left(\begin{array}{cc}
\bar{\Gamma}_{5} & \hat{\mathbf{0}} \\
\hat{\mathbf{0}} & \bar{\Gamma}_{3}
\end{array}\right)-3 i \pi \hat{\mathbf{I}}\right],
$$

where the five-dimensional matrix $\bar{\Gamma}_{5}$ reads

$$
\bar{\Gamma}_{5}=\left(\begin{array}{ccccc}
6 \bar{S} & 0 & 6 \Omega & 0 & 0 \\
0 & 3 \bar{S}+\frac{3}{2} \Lambda & \frac{3}{2} \Omega & 3 \Omega & 0 \\
\frac{3}{4} \Omega & \frac{3}{2} \Omega & 3 \bar{S}+\frac{3}{2} \Lambda & 0 & \frac{9}{4} \Omega \\
0 & \frac{6}{5} \Omega & 0 & 3 \Lambda & \frac{9}{5} \Omega \\
0 & 0 & \frac{2}{3} \Omega & \frac{4}{3} \Omega & 4 \Lambda-2 \bar{S}
\end{array}\right)
$$

and the three-dimensional matrix $\bar{\Gamma}_{3}$ is diagonal,

$$
\bar{\Gamma}_{3}=\operatorname{diag}(3(\bar{S}+\bar{U}), 3(\bar{S}+\bar{T}), 3(\bar{T}+\bar{U})) .
$$

Although both $\tilde{S}_{i j \rightarrow k l, I J}$ and $\tilde{U}_{i \bar{i}}$ are gauge-dependent functions, results for the one-loop $\bar{\Gamma}_{i j \rightarrow k l, I J}^{(1)}$ matrices presented here are gauge-invariant. The gauge dependence in the sum of vertex and self-energy corrections cancels against the gauge dependence of the Drell-Yan anomalous dimension $[19,36]$. The $\tilde{U}_{i \bar{i}}$ soft function, in turn, carries the same gauge dependence as the 
ratio of the incoming jet functions $\left(\tilde{\psi}_{i / i} / \tilde{f}_{i / i}\right)^{2}[21,29]$. In practice, we fix the gauge $A^{0}=0$ to calculate $\Gamma_{i j \rightarrow k l, I J}^{(1)}$ and use $\Gamma_{i \bar{i}}^{(1)}\left(\alpha_{\mathrm{s}}\right)=C_{i} \alpha_{\mathrm{s}} / \pi$ in Eq. (32).

At the production threshold $\beta_{\tilde{g}} \rightarrow 0$ the soft anomalous dimension matrices $\bar{\Gamma}_{q \bar{q} \rightarrow \tilde{g} \tilde{g}}$ and $\bar{\Gamma}_{q \bar{q} \rightarrow \tilde{g} \tilde{g}}$ approach the diagonal form. The off-diagonal terms, proportional to $\Omega$, vanish like $\beta_{\tilde{g}}$ for $\beta_{\tilde{g}} \rightarrow 0$ and thus may be neglected. Using Eq. (43) we obtain

$$
\begin{aligned}
& \left\{D_{q \bar{q} \rightarrow \tilde{g} \tilde{g}, I}^{(1)}\right\}=\{0,-3,-3\} \\
& \left\{D_{g g \rightarrow \tilde{g} \tilde{g}, I}^{(1)}\right\}=\{0,-3,-3,-6,-8 ;-3,-3,-6\},
\end{aligned}
$$

where the index $I$ indicates a colour channel, defined by the base tensor $c_{I}^{q, \tilde{g}}$ and $c_{I}^{g, \tilde{g}}$ as in (5) and (8), correspondingly. Note that the values of the $D^{(1)}$-coefficients are the negative values of the quadratic Casimir operators for the SU(3) representations for the outgoing state. This agrees with the physical picture of the soft gluon radiation from the total colour charge of the heavy-particle pair produced at threshold [23].

\subsection{Squark-antisquark production}

For completeness, we also list here the NLL coefficients $D_{i j \rightarrow \tilde{q} \overline{\tilde{q}}, I}^{(1)}$ which we need for our numerical calculations. They have been first obtained in the calculation of resummed cross sections for the heavy quark production and read [19, 23]

$$
\begin{aligned}
& \left\{D_{q \bar{q} \rightarrow \tilde{q} \overline{\tilde{q}}, I}^{(1)}\right\}=\{0,-3\} \\
& \left\{D_{g g \rightarrow \tilde{q} \tilde{\tilde{q}}, I}^{(1)}\right\}=\{0,-3,-3\}
\end{aligned}
$$

with the index $I$ indicating the corresponding tensors in the $s$-channel colour basis $c_{I}^{q, \tilde{q}}$ and $c_{I}^{g, \tilde{q}}$, given in (4) and (5).

\subsection{Checks of analytical results}

The computational framework applied here has been tested by re-deriving the known results for the one-loop soft anomalous matrices for the pair-production of massive particles. We have reproduced the results for the heavy quark production [19] both in the $q \bar{q}$ and in the $g g$ channel.

Another test of our calculations has been based on a comparison between expansion of the resummed cross section, Eq. (40), and the Mellin moments of the NLO corrections taken in the threshold limit. The analytic form of the NLO corrections in the threshold limit in momentum space is known for all squark and gluino production processes [6]. More precisely, for each of the partonic processes, $q_{i} \bar{q}_{j} \rightarrow \tilde{q} \overline{\tilde{q}}, g g \rightarrow \tilde{q} \overline{\tilde{q}}, q \bar{q} \rightarrow \tilde{g} \tilde{g}$, and $g g \rightarrow \tilde{g} \tilde{g}$, we have extracted terms with $\log ^{2} N$ and $\log N$ from the $\mathcal{O}\left(\alpha_{\mathrm{s}}\right)$ expansion of the corresponding resummed formula. In each case the result has been compared with the collection of terms logarithmic in $N$ in the Mellin 
transform of the $\mathcal{O}\left(\alpha_{\mathrm{s}}\right)$ correction taken in the large $N$ limit. The contributions enhanced by double logarithms, i.e. $\mathcal{O}\left(\alpha_{\mathrm{s}} \log ^{2} N\right)$, depend only on the colour charges of the incoming partons and thus do not provide a check of the soft anomalous dimension matrices. A non-trivial crosscheck is, however, provided by the contributions to the NLO correction with single logarithms, $\mathcal{O}\left(\alpha_{s} \log N\right)$, that are sensitive to the eigenvalues of the $\bar{\Gamma}$ matrices. Our result for the resummed cross section, Eq. (40), agree in this way with results of Ref. [6].

\section{Resummation of leading Coulomb corrections}

Important higher order corrections to cross sections for production of coloured particles come from multiple exchanges of Coulomb gluons between the produced particles. This type of corrections should be then taken into account to all orders $[17,37,38]$. In the threshold limit, the Coulomb corrections are enhanced by the inverse powers of $\beta$. At $n$-th order in perturbation

theory the leading corrections are of the form $C_{\mathrm{Coul}}^{(n)} \alpha_{\mathrm{s}}^{n} / \beta^{n}$. These leading contributions can be summed to all orders using [37]

$$
\hat{\sigma}_{i j \rightarrow k l, I}^{(C)}=\sigma_{i j \rightarrow k l, I}^{(0)} \Delta^{(C)}\left(\frac{\pi \alpha_{s}}{\beta} \kappa_{i j \rightarrow k l, I}\right),
$$

where

$$
\Delta^{(C)}(z)=\frac{z}{\exp (z)-1}
$$

and $\beta=\beta_{\tilde{q}}$ for $\tilde{q} \tilde{\tilde{q}}$ production, $\beta=\beta_{\tilde{g}}$ for $\tilde{g} \tilde{g}$ production. For the processes of interest, the $\kappa$ coefficients, calculated in the set of colour basis introduced in Section 2, are given by

$$
\begin{aligned}
& \kappa_{q \bar{q} \rightarrow \tilde{q} \tilde{q}, I}=\left(-\frac{4}{3}, \frac{1}{6}\right), \\
& \kappa_{g g \rightarrow \tilde{q} \tilde{q}, I}=\left(-\frac{4}{3}, \frac{1}{6}, \frac{1}{6}\right), \\
& \kappa_{q \bar{q} \rightarrow \tilde{g} \tilde{g}, I}=\left(-3,-\frac{3}{2},-\frac{3}{2}\right), \\
& \kappa_{g g \rightarrow \tilde{g} \tilde{g}, I}=\left(-3,-\frac{3}{2},-\frac{3}{2}, 0,1 ;-\frac{3}{2},-\frac{3}{2}, 0\right) .
\end{aligned}
$$

The $\mathcal{O}\left(\alpha_{s}\right)$ Coulomb correction is a part of the full NLO result. Since we are interested in corrections above NLO, we subtract it from $\hat{\sigma}_{i j \rightarrow k l, I}^{(C)}$. In this way we define the correction $\delta \hat{\sigma}_{i j \rightarrow k l, I}^{(C)}$ due to leading (in terms of powers of $1 / \beta$ ) Coulomb contributions above NLO

$$
\delta \hat{\sigma}_{i j \rightarrow k l, I}^{(C)}=\hat{\sigma}_{i j \rightarrow k l, I}^{(C)}-\left.\hat{\sigma}_{i j \rightarrow k l, I}^{(C)}\right|_{(\mathrm{NLO})} .
$$

The corresponding Coulomb correction at the hadronic level then reads

$\delta \sigma_{h_{1} h_{2} \rightarrow k l}^{(C)}\left(\rho,\left\{m^{2}\right\}\right)=\sum_{i, j ; I} \int d x_{1} d x_{2} d \hat{\rho} \delta\left(\hat{\rho}-\frac{\rho}{x_{1} x_{2}}\right) f_{i}\left(x_{1}, \mu\right) f_{j}\left(x_{2}, \mu\right) \delta \hat{\sigma}_{i j \rightarrow k l, I}^{(C)}\left(\hat{\rho},\left\{m^{2}\right\}, \mu^{2}\right)$. 


\section{Predictions for squarks and gluino production at the LHC}

We investigate in detail the effect of the soft gluon corrections on the cross sections for two sparticle production processes at the LHC, $p p \rightarrow \tilde{g} \tilde{g}$ and $p p \rightarrow \tilde{q} \overline{\tilde{q}}$, at $\sqrt{S}=14 \mathrm{TeV}$. The main results obtained in this section are the resummation-improved total cross sections. We also study the effect of the resummed leading Coulomb corrections to the total cross sections. Moreover, we present a detailed analysis of the soft gluon corrections in partonic channels of hadronic processes, including also the dependence of soft gluon effects on the colour structure of the hard matrix element. All numerical calculations were performed using two independent computer codes.

\subsection{Inversion and matching}

The resummation-improved cross sections are obtained through matching the NLL resummed expressions with the full NLO cross sections,

$$
\begin{aligned}
\sigma_{h_{1} h_{2} \rightarrow k l}^{(\text {match })}\left(\rho,\left\{m^{2}\right\}\right)= & \sigma_{h_{1} h_{2} \rightarrow k l}^{(\mathrm{NLO})}\left(\rho,\left\{m^{2}\right\}\right) \\
& +\sum_{i, j=q, \bar{q}, g} \int_{\mathrm{CT}} \rho^{-N} \tilde{f}_{i / h_{1}}\left(N+1, \mu^{2}\right) \tilde{f}_{j / h_{2}}\left(N+1, \mu^{2}\right) \\
\times & {\left[\tilde{\sigma}_{i j \rightarrow k l}^{(\mathrm{res})}\left(N,\left\{m^{2}\right\}\right)-\left.\tilde{\sigma}_{i j \rightarrow k l}^{(\mathrm{res})}\left(N,\left\{m^{2}\right\}\right)\right|_{(\mathrm{NLO})}\right], }
\end{aligned}
$$

where $\tilde{\sigma}_{i j \rightarrow k l}^{(\mathrm{res})}$ is given through Eq. (40) together with Eq. (23), and $\left.\tilde{\sigma}_{i j \rightarrow k l}^{(\mathrm{res})}\right|_{(\mathrm{NLO})}$ represents its perturbative expansion truncated at the order of $\alpha_{\mathrm{s}}$ associated with the NLO correction.

The inverse Mellin transform (58) is evaluated numerically using a contour CT in the complex- $N$ space according to the "Minimal Prescription" method developed in Ref. [27]. More specifically, we use a contour parameterised by a parameter $\chi, N=C_{0}+\chi \exp ( \pm i \phi)$, as described in $[39,40]$. In order to be able to use available parameterisations of parton distribution functions in $x$-space we apply the method introduced in [39]. The NLO cross sections are evaluated using Prospino [41], the numerical package based on calculations employing the $\overline{\mathrm{MS}}$ renormalisation and factorisation schemes.

\subsection{Numerical results}

In the phenomenological analysis we consider a wide range of gluino and squark masses. Left- and right-handed squarks of all flavours are assumed to be mass degenerate. For the $\tilde{g} \tilde{g}$ production we vary the gluino mass, $m_{\tilde{g}}$, between $200 \mathrm{GeV}$ and $2 \mathrm{TeV}$. Similarly, for the $\tilde{q} \overline{\tilde{q}}$ production we take ${ }^{2} 200 \mathrm{GeV}<m_{\tilde{q}}<2 \mathrm{TeV}$. We present the results for a fixed ratio of gluino and squark

\footnotetext{
${ }^{2}$ For the highest masses considered here, the experimental exploration at the LHC will require luminosities of $\mathcal{O}\left(100 \mathrm{fb}^{-1}\right)$.
} 
masses, $r=\frac{m_{\tilde{g}}}{m_{\tilde{q}}}$, and choose the following values $r=0.5,0.81 .21 .6,2.0$. The $\tilde{q} \tilde{\tilde{q}}$ cross section accounts for production of all $\tilde{q} \tilde{\tilde{q}}$ flavour combinations apart from the ones with scalar top particles.

For most of the phenomenological results, we use the CTEQ6M [42] parameterisation of parton distribution functions (pdfs). In addition, we give the total cross sections and study their scale dependence for the MSTW parameterisation [43] of the pdfs. Unless explicitly specified otherwise, the CTEQ6 pdfs are applied. In both sets of pdfs the usual assumption of five massless quark flavours active at large scales is made. Consequently, in the NLO and NLL calculations we use the two-loop $\overline{\mathrm{MS}}$ QCD running coupling constant $\alpha_{\mathrm{s}}$ with $n_{\mathrm{f}}=5$. We also show some results obtained at the LO accuracy using CTEQ6L1 parameterisation of the pdfs and the one-loop running coupling constant with 5 flavors. For all parameterisations we consistently use the corresponding default values of $\Lambda^{(5)}$. The effects due to virtual top quarks and virtual sparticles in the running of $\alpha_{\mathrm{s}}$ and in the evolution of pdfs are thus not included in our predictions. However, the value of the top mass $m_{t}=175 \mathrm{GeV}$ enters the matched NLL cross sections through the NLO corrections.

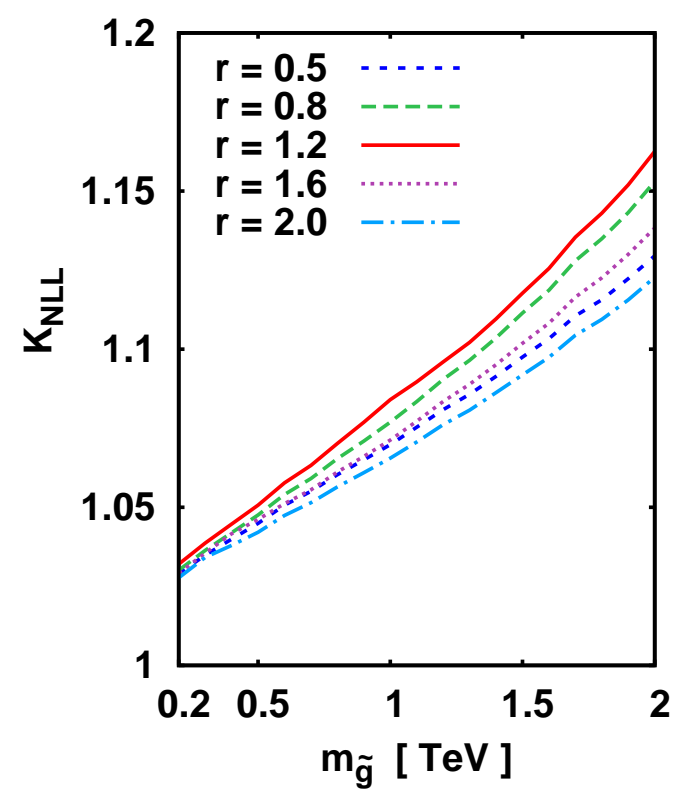

a)

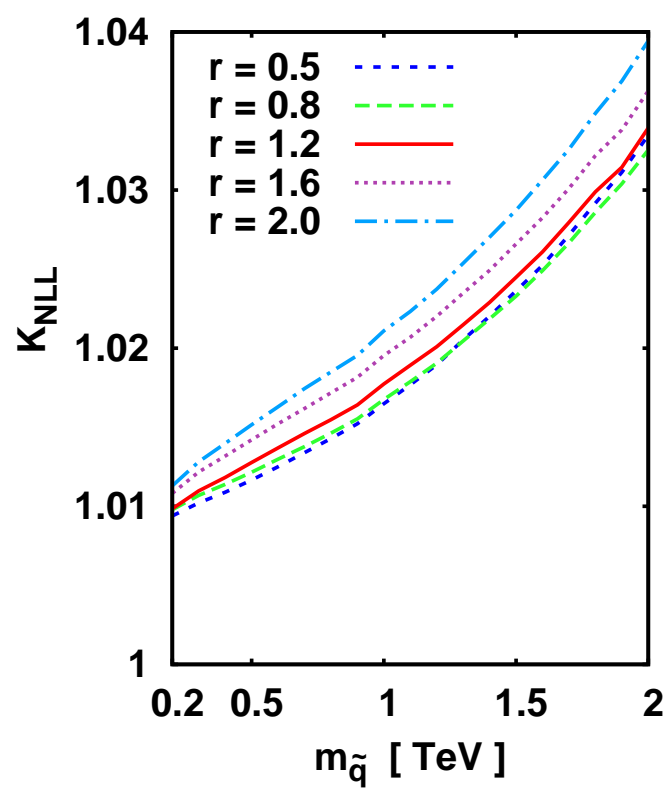

b)

Figure 2: The NLL K-factor, $K_{\mathrm{NLL}}$, for the $\tilde{g} \tilde{g}(a)$ and the $\tilde{q} \overline{\tilde{q}}(b)$ total production cross section at the LHC as a function of gluino and squark mass, respectively; $r=m_{\tilde{g}} / m_{\tilde{q}}$.

In Fig. 2 we present the relative enhancement of the NLO total cross sections due to soft gluon resummation, $K_{\mathrm{NLL}} \equiv \sigma^{(\text {match })} / \sigma^{\mathrm{NLO}}$. The NLL $K$-factors are shown for $\tilde{g} \tilde{g}$ and $\tilde{q} \overline{\tilde{q}}$ production cross sections at the LHC, in Fig. 2a and Fig. 2b, respectively. In the plots we set the scales $\mu_{F}=\mu_{R}=\mu_{0}$, where $\mu_{0}=m_{\tilde{g}}\left(\mu_{0}=m_{\tilde{q}}\right)$ for the $\tilde{g} \tilde{g}$ production (the $\tilde{q} \overline{\tilde{q}}$ production). 
The NLO CTEQ6M pdfs are used. $K_{\text {NLL }}$ grows with the final-state mass and depends on the mass ratio $r$ in a moderate way. The relative correction $K_{\mathrm{NLL}}-1$ reaches $16 \%(8 \%)$ for the $\tilde{g} \tilde{g}$ production with $r=1.2$ and $m_{\tilde{g}}=2 \mathrm{TeV}(1 \mathrm{TeV})$, and $4 \%(2 \%)$ for the $\tilde{q} \overline{\tilde{q}}$ production with $r=2$ and $m_{\tilde{q}}=2 \mathrm{TeV}(1 \mathrm{TeV})$. The stronger effect found in the $\tilde{g} \tilde{g}$ production follows from the dominance of the $g g \rightarrow \tilde{g} \tilde{g}$ channel and hence larger colour factors. It comes from the fact that the size of the soft-collinear radiative factor $\Delta_{i}$ increases with higher colour charge of the incoming parton. Similarly, the size of the soft non-collinear gluon corrections increases with the total colour charge of the final state, which may be the highest in the $g g \rightarrow \tilde{g} \tilde{g}$ case.

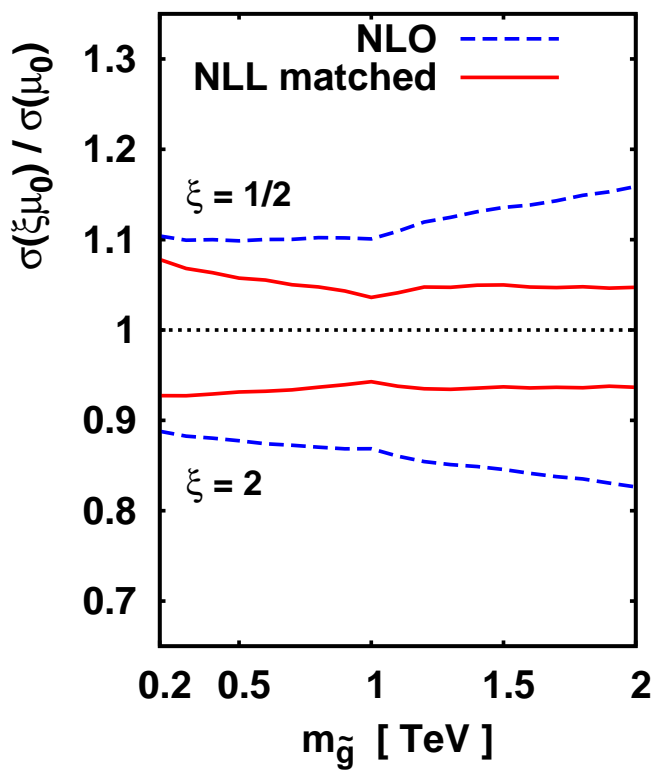

a)

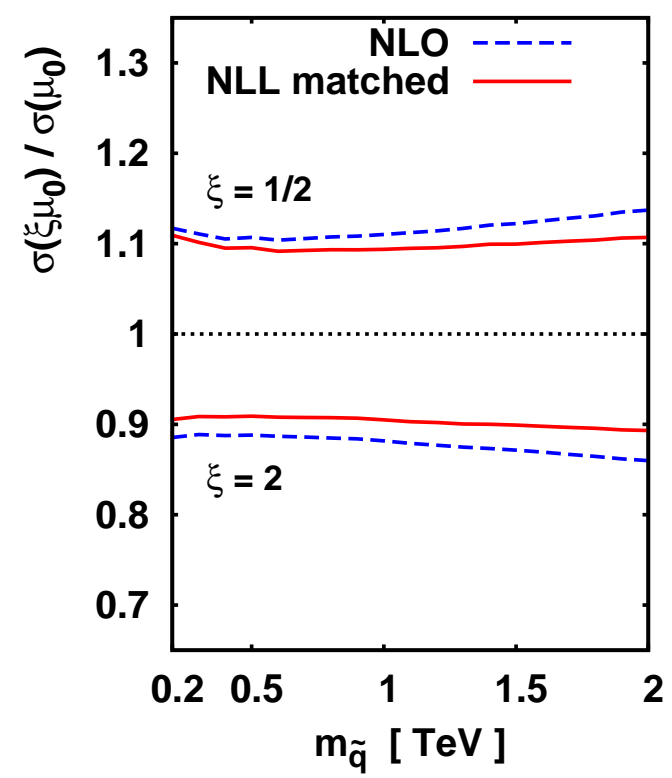

b)

Figure 3: Scale dependence of the total $\tilde{g} \tilde{g}$ (a) and $\tilde{q} \overline{\tilde{q}}$ (b) production cross section at the LHC (see the text for explanation).

We also investigate the dependence of the matched NLL cross section on the values of factorisation and renormalisation scales, in comparison to the NLO cross section. To illustrate our results we choose $\mu=\mu_{F}=\mu_{R}$ and $r=1$.2. In Fig. 3a and Fig. 3b we plot the ratios $\sigma^{\mathrm{NLO}}\left(\mu=\xi \mu_{0}\right) / \sigma^{\mathrm{NLO}}\left(\mu=\mu_{0}\right)$ and $\sigma^{(\text {match })}\left(\mu=\xi \mu_{0}\right) / \sigma^{(\text {match })}\left(\mu=\mu_{0}\right)$, obtained by varying $\xi$ between $\xi=1 / 2$ and $\xi=2$. Due to resummation the scale sensitivity of the $\tilde{g} \tilde{g}$ production cross section reduces significantly, by a factor of $\sim 3(\sim 2)$ at $m_{\tilde{g}}=2 \mathrm{TeV}\left(m_{\tilde{g}}=1 \mathrm{TeV}\right)$. At $m_{\tilde{g}}>1 \mathrm{TeV}$ the theoretical error of the matched NLL $\tilde{g} \tilde{g}$ cross section, defined by changing the scale $\mu=\mu_{F}=\mu_{R}$ around $\mu_{0}=m_{\tilde{g}}$ by a factor of 2 , is around $5 \%$. In the case of the $\tilde{q} \overline{\tilde{q}}$ production the reduction of the scale dependence due to including soft gluon corrections in the theoretical predictions is moderate.

The dependence on the pdf parameterisation of the NLL $K$-factors for $\tilde{g} \tilde{g}$ and $\tilde{q} \overline{\tilde{q}}$ production 


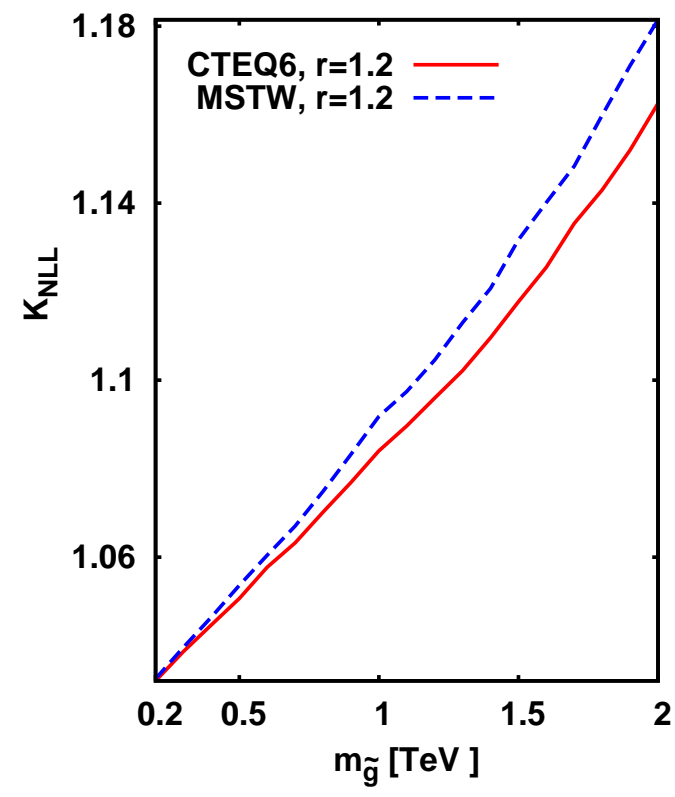

a)

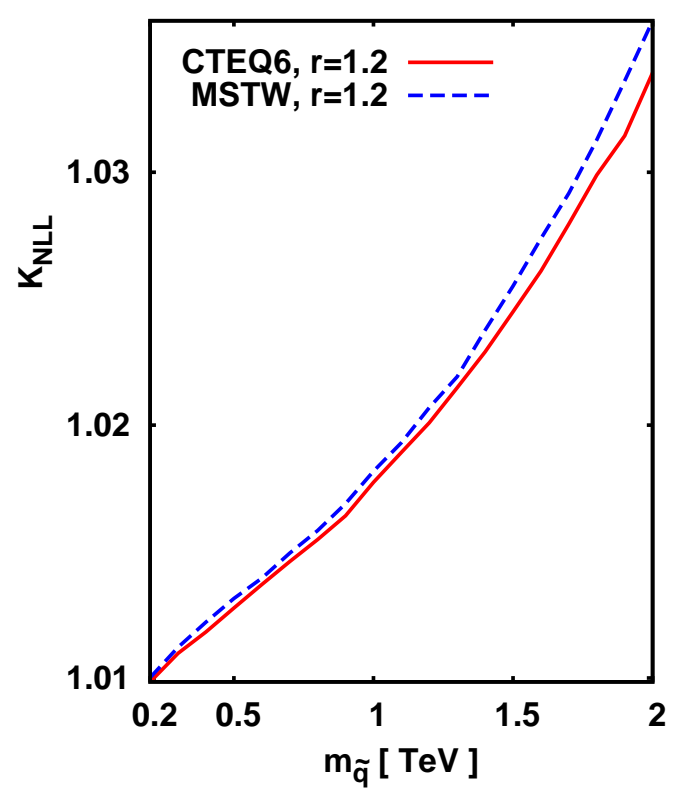

b)

Figure 4: The relative $N L L$ correction $K_{\mathrm{NLL}}$ for the $\tilde{g} \tilde{g}(a)$ and the $\tilde{q} \tilde{\tilde{q}}$ (b) total production cross section at the LHC as a function of gluino and squark mass, respectively; $r=m_{\tilde{g}} / m_{\tilde{q}}$. The continuous lines correspond to the CTEQ6M pdfs and the dashed lines to the MSTW pdfs.

at the LHC is presented in Fig. 4a and Fig. 4b, respectively. The MSTW pdfs, shown with the dashed lines, lead to slightly larger NLL $K$-factors than the CTEQ6M pdfs. In this figure we choose $r=m_{\tilde{g}} / m_{\tilde{q}}=1.2$. The difference between the $K$-factors for the two parameterisations is moderate but it grows with increasing mass of the produced sparticles. It is expected since for larger masses of sparticles larger values of the factorisation scale $\mu_{F}$ and of the momentum fraction $x$ are probed. For larger values of $\mu_{F}$ and $x$ the pdfs are currently not so well constrained, see e.g. the discussion of the uncertainties of the pdfs given in [43].

Next, we analyse the combined dependence of the NLO and the matched NLL total cross sections on the factorisation and the renormalisation scale and on the type of the pdfs. The results are shown in Fig. 5 and Fig. 6 for the $\tilde{g} \tilde{g}$ and $\tilde{q} \tilde{\tilde{q}}$ production, respectively. Again, the renormalisation and factorisation scales are assumed to be equal, $\mu_{F}=\mu_{R}=\mu=\xi \mu_{0}$, where the scale $\mu_{0}$ is set equal to the average mass of the produced particles. The parameter $\xi$ is varied between 0.2 and 5. In Fig. 5a, Fig. 5b and Fig. 5c (Fig. 6a, Fig. 6b and Fig. 6c) the gluino (squark) mass, $m_{\tilde{g}}\left(m_{\tilde{q}}\right)$, takes values of $0.5,1$ and $2 \mathrm{TeV}$, correspondingly, and $r=m_{\tilde{g}} / m_{\tilde{q}}=1.2$.

The theoretical uncertainty of the $p p \rightarrow \tilde{g} \tilde{g}$ cross section due to differences in the parameterisations of the pdfs may be read out from Fig. 5. At the NLO the uncertainty is given by the difference between the dotted (CTEQ6M) and the dash-dotted (MSTW) lines and at the NLL by the difference between the continuous (CTEQ6M) and the dashed (MSTW) lines. The 


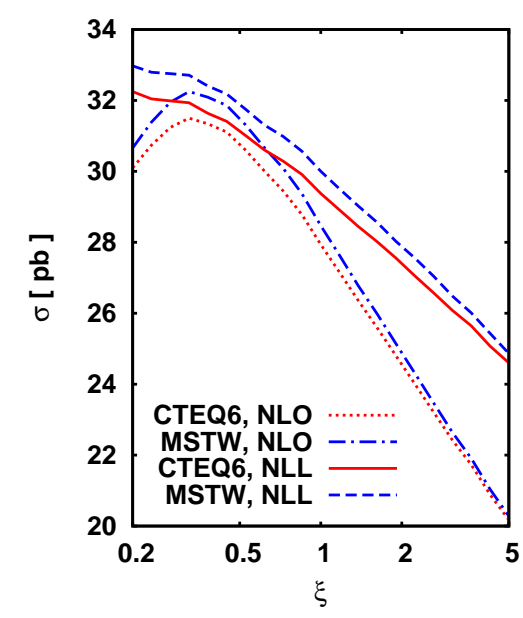

a)

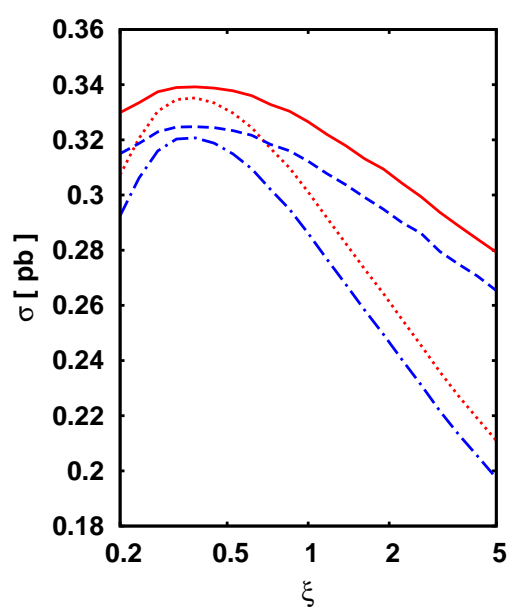

b)

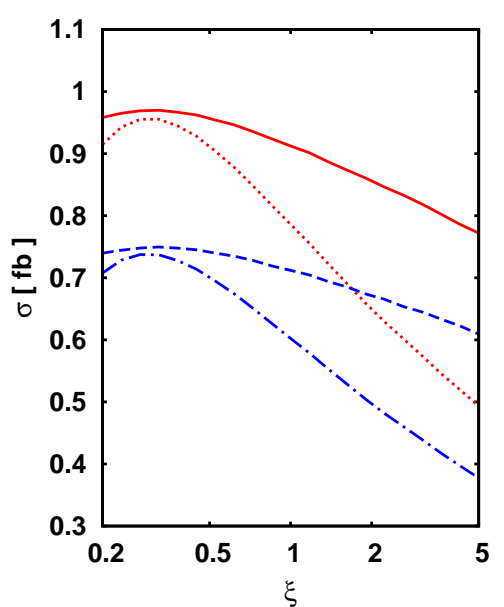

c)

Figure 5: The dependence of the total cross section $p p \rightarrow \tilde{g} \tilde{g}$ on the renormalisation and factorisation scale, $\mu=\mu_{R}=\mu_{F}=\xi m_{\tilde{g}}$, for $r=m_{\tilde{g}} / m_{\tilde{q}}=1.2$ and: (a) $m_{\tilde{g}}=0.5 \mathrm{TeV}$, (b) $m_{\tilde{g}}=1 \mathrm{TeV}$ and (c) $m_{\tilde{g}}=2 \mathrm{TeV}$. The four lines in each plot correspond to the NLO and NLL cross section, each one evaluated with CTEQ6M and MSTW pdfs, see the legend.

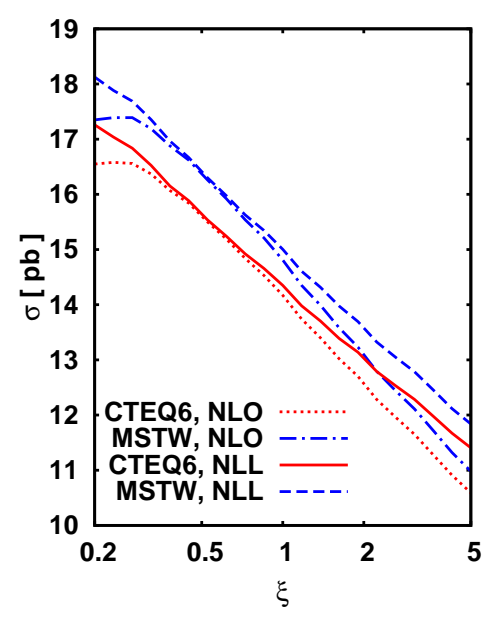

a)

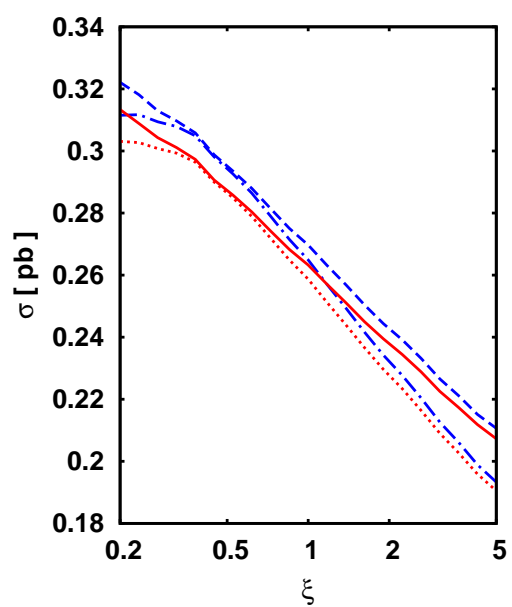

b)

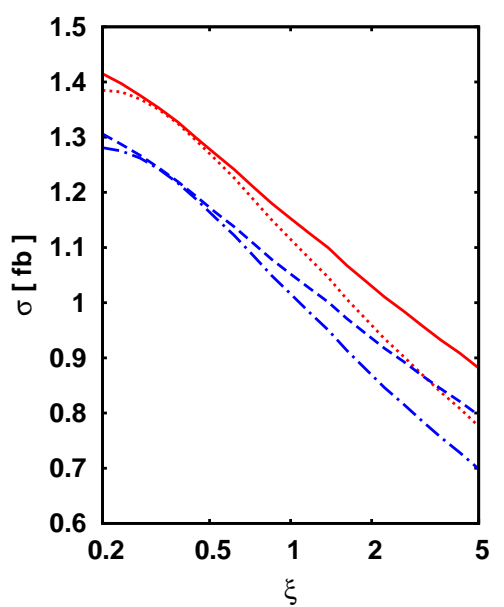

c)

Figure 6: The dependence of the total cross section $p p \rightarrow \tilde{q} \overline{\tilde{q}}$ on the renormalisation and factorisation scale, $\mu=\mu_{R}=\mu_{F}=\xi m_{\tilde{q}}$, for $r=m_{\tilde{g}} / m_{\tilde{q}}=1.2$ and: (a) $m_{\tilde{q}}=0.5 \mathrm{TeV}$, (b) $m_{\tilde{q}}=1 \mathrm{TeV}$ and (c) $m_{\tilde{q}}=2 \mathrm{TeV}$. The four lines in each plot correspond to the NLO and NLL cross section, each one evaluated with CTEQ6M and MSTW pdfs, see the legend. 
absolute difference between the CTEQ6 curves and the MSTW curves is rather similar at the NLO and at the NLL accuracy. It is clearly visible in Fig. 5 that the uncertainty introduced by the pdfs grows with the increasing gluino mass. In particular, for $m_{\tilde{g}}=0.5 \mathrm{TeV}$ this uncertainty is smaller than $3 \%$, for $m_{\tilde{g}}=1 \mathrm{TeV}$ it is smaller than $5 \%$, and for $m_{\tilde{g}}=2 \mathrm{TeV}$ it reaches already about $25 \%$. We rephrase that the probable reason for the strong dependence of the cross sections on the pdfs at large gluino mass is the fact that the currently available pdfs are poorly constrained at large scales and at large parton $x$. This uncertainty should be, however, substantially reduced after high $p_{T}$ jet measurements are perfomed at the LHC. As seen in Fig. 5 , the variation of the $p p \rightarrow \tilde{g} \tilde{g}$ cross section with the scale $\mu=\xi m_{\tilde{g}}$ is substantially reduced after inclusion of the soft gluon resummation over the whole $\xi$ range.

The case of squark-antisquark production is illustrated in Fig. 6. Clearly, for $\tilde{q} \overline{\tilde{q}}$ production, the soft gluon resummation introduces much smaller reduction in the scale dependence over the whole $\xi$ range than it was in the case of the $\tilde{g} \tilde{g}$ production. The relative uncertainty of the $p p \rightarrow \tilde{q} \overline{\tilde{q}}$ cross section due to the choice of the pdfs varies from about $3 \%$ at $m_{\tilde{q}}=1 \mathrm{TeV}$ by about $5 \%$ at $m_{\tilde{q}}=0.5 \mathrm{TeV}$ to about $8 \%$ at $m_{\tilde{q}}=2 \mathrm{TeV}$. Again, this uncertainty should be reduced after suitable measurements at the LHC are performed.

We also study the corrections to the cross sections for $p p \rightarrow \tilde{g} \tilde{g}$ and $p p \rightarrow \tilde{q} \overline{\tilde{q}}$ coming from the leading Coulomb exchanges beyond the NLO accuracy. We define the corresponding Coulomb $K$-factor

$$
K_{\mathrm{Coul}}=\frac{\sigma_{h_{1} h_{2} \rightarrow k l}^{(\mathrm{NLO})}\left(\rho,\left\{m^{2}\right\}\right)+\delta \sigma_{h_{1} h_{2} \rightarrow k l}^{(C)}\left(\rho,\left\{m^{2}\right\}\right)}{\sigma_{h_{1} h_{2} \rightarrow k l}^{(\mathrm{NLO})}\left(\rho,\left\{m^{2}\right\}\right)},
$$

where we use Eq. (57) to calculate $\delta \sigma_{h_{1} h_{2} \rightarrow k l}^{(C)}\left(\rho,\left\{m^{2}\right\}\right)$ with the two-loop $\alpha_{\text {s }}$ taken at the scale $\mu^{2}$. The obtained numerical results for the relative Coulomb correction, $K_{\text {Coul }}-1$, to the $\tilde{g} \tilde{g}$ and $\tilde{q} \overline{\tilde{q}}$ cross sections are shown in Fig. 7a and Fig. 7b, respectively, and compared to the corresponding relative soft gluon correction, $K_{\mathrm{NLL}}-1$. For the gluino pair-production we find that the Coulomb $K$-factor exhibits only weak dependence on the gluino and squark masses. In more detail, $K_{\text {Coul }}-1$ takes values between $5 \%$ and $6 \%$. In fact, the Coulomb corrections exceed the soft gluon corrections for $m_{\tilde{g}}<0.5 \mathrm{TeV}$ and are relatively important up to $m_{\tilde{g}}=2 \mathrm{TeV}$. For the $\tilde{q} \overline{\tilde{q}}$ production $K_{\text {Coul }}-1$ takes the values between $2 \%$ and $3.5 \%$, depending on $m_{\tilde{q}}$ and $m_{\tilde{g}}$. The importance of the leading Coulomb corrections in $\tilde{q} \overline{\tilde{q}}$ production can be seen in Fig. $7 \mathrm{~b}$.

The NLL soft gluon corrections and the Coulomb corrections beyond the NLO approximation (57) can be combined additively. We define the $K$-factor that accounts for both types of corrections in the following way:

$$
K_{\mathrm{NLL}+\mathrm{Coul}}=\frac{\sigma_{h_{1} h_{2} \rightarrow k l}^{(\text {match })}\left(\rho, m^{2},\left\{\mu^{2}\right\}\right)+\delta \sigma_{h_{1} h_{2} \rightarrow k l}^{(C)}\left(\rho, m^{2},\left\{\mu^{2}\right\}\right)}{\sigma_{h_{1} h_{2} \rightarrow k l}^{(\mathrm{NLO})}\left(\rho, m^{2},\left\{\mu^{2}\right\}\right)} .
$$




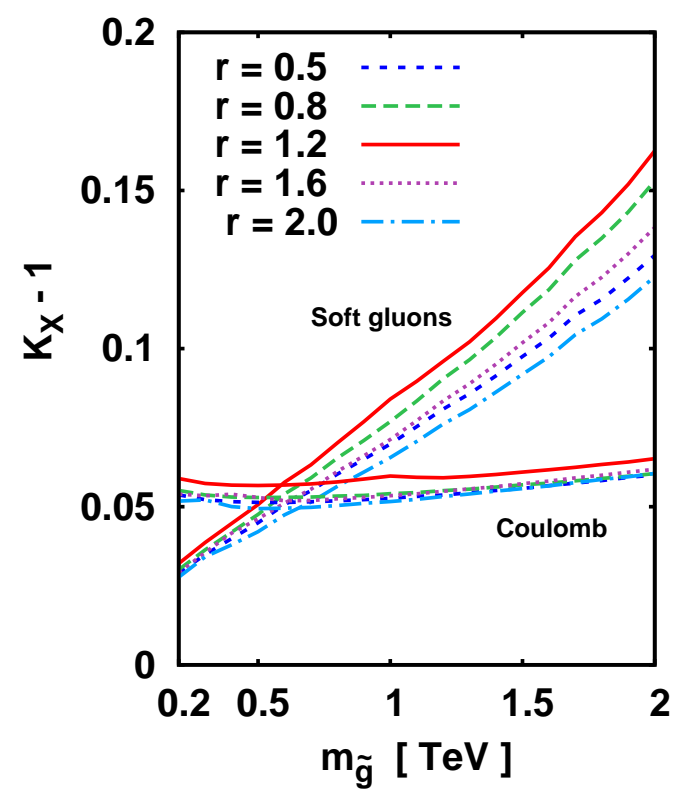

a)

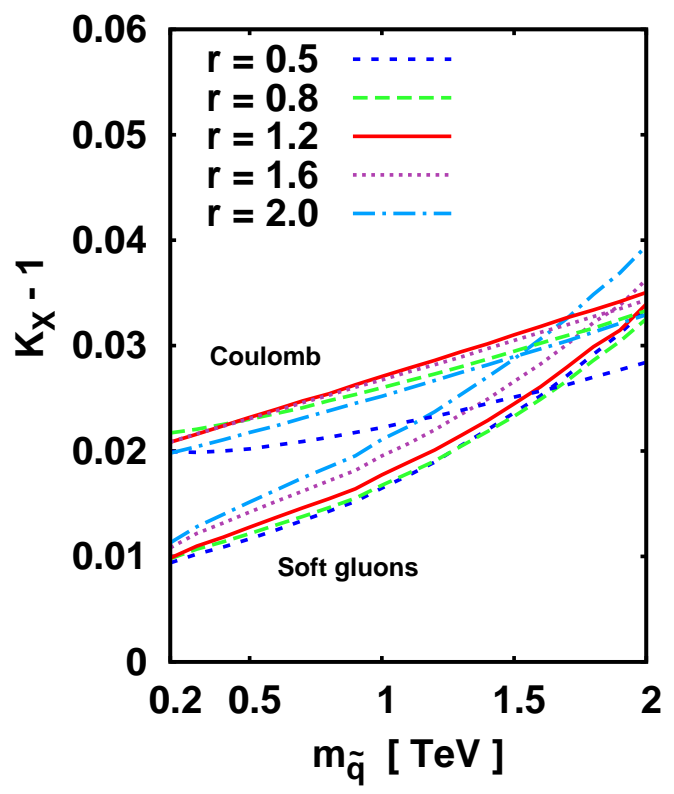

b)

Figure 7: The relative corrections, $K_{\mathrm{NLL}}-1$ and $K_{\mathrm{Coul}}-1$, to the $N L O$ cross sections for the $\tilde{g} \tilde{g}$ (a) and the $\tilde{q} \bar{q}$ (b) production at the LHC as a function of gluino and squark mass, respectively; $r=m_{\tilde{g}} / m_{\tilde{q}}$.

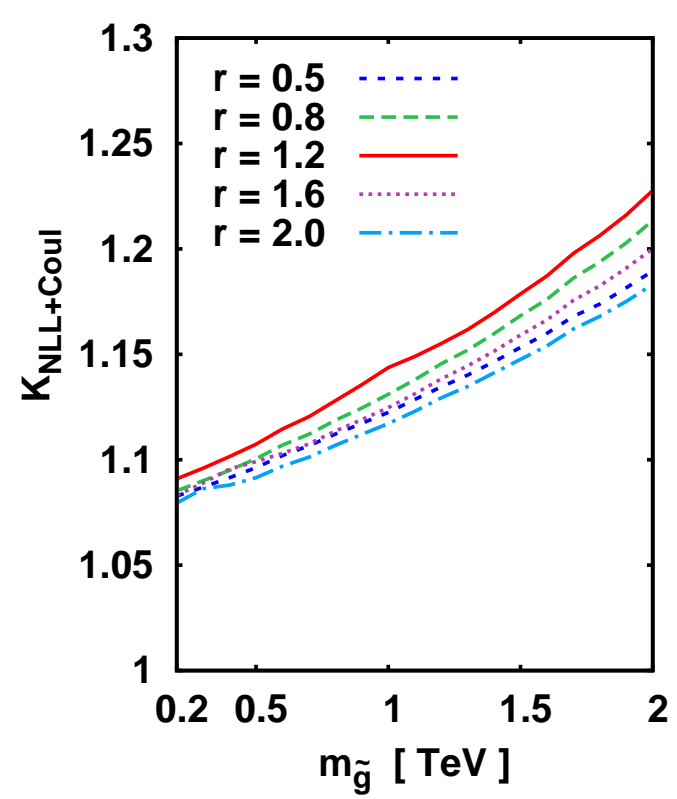

a)

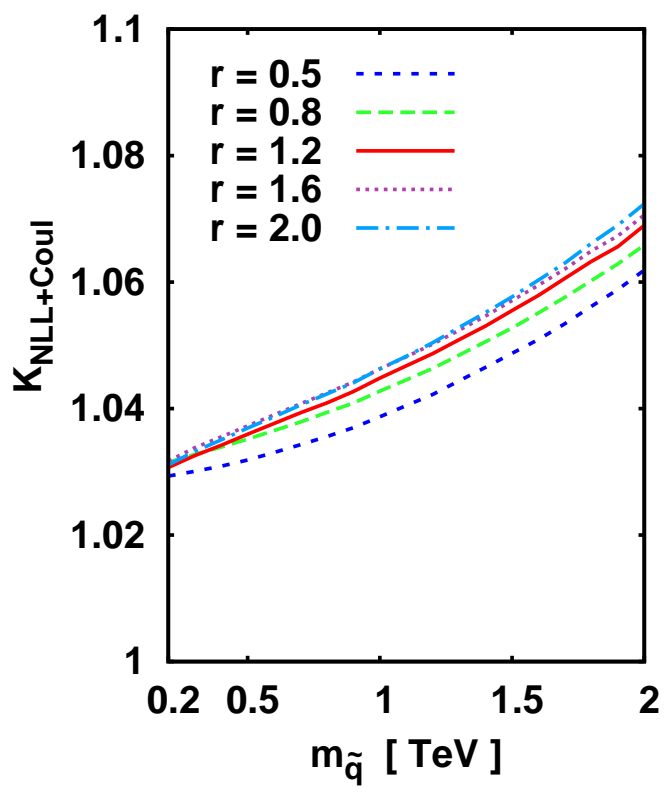

b)

Figure 8: The $K$-factors, $K_{\mathrm{NLL}+\mathrm{Coul}}$ for the $\tilde{g} \tilde{g}(a)$ and the $\tilde{q} \overline{\tilde{q}}$ (b) total production cross sections at the LHC as a function of gluino and squark mass, respectively; $r=m_{\tilde{g}} / m_{\tilde{q}}$. 
The obtained $K$-factors, $K_{\mathrm{NLL}+\mathrm{Coul}}$, in the $\tilde{g} \tilde{g}$ and $\tilde{q} \tilde{\tilde{q}}$ production at the LHC are shown in Fig. $8 \mathrm{a}$ and Fig. 8b, respectively. The results shown in Fig. 8 are our most complete estimates of the higher order QCD corrections in these processes.

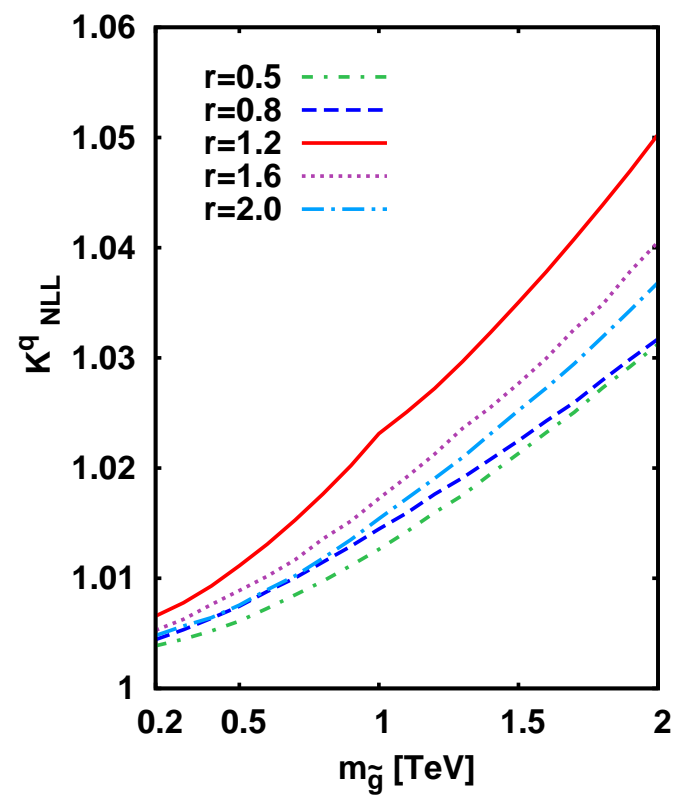

a)

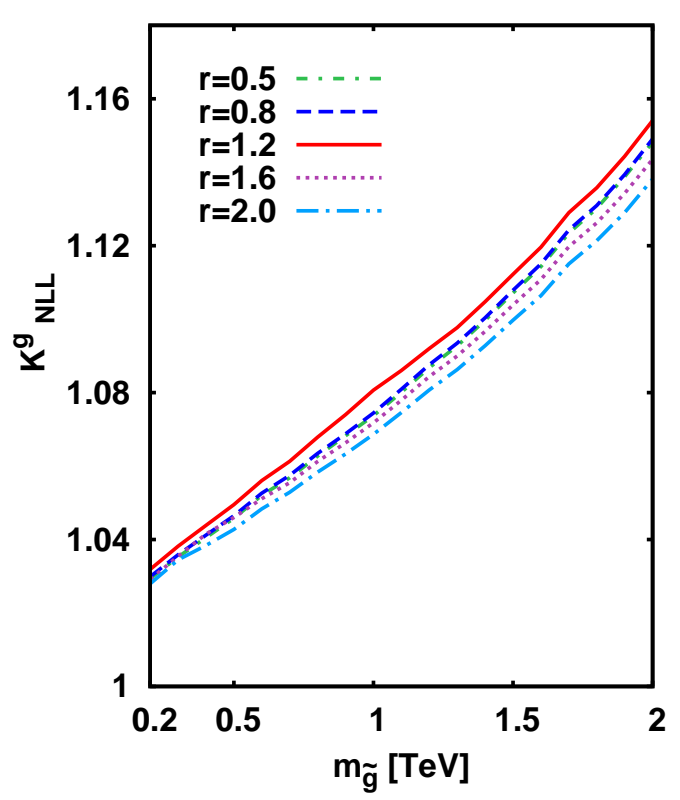

b)

Figure 9: The $K$-factor, $K_{\mathrm{NLL}}^{i}$, for the partonic channels in the hadronic process $p p \rightarrow \tilde{g} \tilde{g}:$ (a) $q \bar{q} \rightarrow \tilde{g} \tilde{g}$ and (b) $g g \rightarrow \tilde{g} \tilde{g}$.

It is interesting to investigate in more detail the soft gluon corrections in partonic subchannels of the $\tilde{g} \tilde{g}$ and $\tilde{q} \overline{\tilde{q}}$ hadroproduction at the LHC. For this purpose we define the NLL $K$-factors restricted to the subprocesses occurring through the $q \bar{q}$ and the $g g$ partonic collision, i.e. we define $K_{\mathrm{NLL}}^{q}=\sigma_{p p \rightarrow q \bar{q} \rightarrow k l}^{\text {(match })} / \sigma_{p p \rightarrow q \bar{q} \rightarrow k l}^{\mathrm{NLO}}$ and $K_{\mathrm{NLL}}^{g}=\sigma_{p p \rightarrow g g \rightarrow k l}^{(\text {match })} / \sigma_{p p \rightarrow g g \rightarrow k l}^{\mathrm{NLO}}$, where, as usual, $k l=\tilde{g} \tilde{g}$ or $k l=\tilde{q} \overline{\tilde{q}}$. The results for $K_{\mathrm{NLL}}^{i}$ in the $\tilde{g} \tilde{g}$ and in the $\tilde{q} \tilde{\tilde{q}}$ production are given in Fig. 9 and Fig. 10, respectively. The NLL $K$-factors grow with increasing masses of the produced particles in all cases. This is expected since the importance of the threshold logarithms strengthens with the higher masses of the produced particles.

It is clearly visible from Fig. 9 and Fig. 10 that the size of the soft gluon corrections increases with higher colour charges of all particles involved in the partonic reaction. The NLL correction for the $g g$ mediated processes (see Fig. 9a and Fig. 10a) is much larger than the NLL correction for the $q \bar{q}$ mediated processes (Fig. $9 \mathrm{~b}$ and Fig. 10b), $K_{\mathrm{NLL}}^{g}-1>K_{\mathrm{NLL}}^{q}-1$, both for the $\tilde{g} \tilde{g}$ and in $\tilde{q} \overline{\tilde{q}}$ production. More specifically, $K_{\mathrm{NLL}}^{g}-1$ is $3-5$ times larger than $K_{\mathrm{NLL}}^{q}-1$, depending on the final state particles and their masses. By comparing the $K$-factors with the same partonic initial state in the production of $\tilde{g} \tilde{g}$ and $\tilde{q} \overline{\tilde{q}}$ (i.e. by comparing Fig. 9a with Fig. 10a and Fig. 9b with Fig. 10b) one finds that the soft gluon effects are larger for $\tilde{g} \tilde{g}$ production than for $\tilde{q} \overline{\tilde{q}}$ 


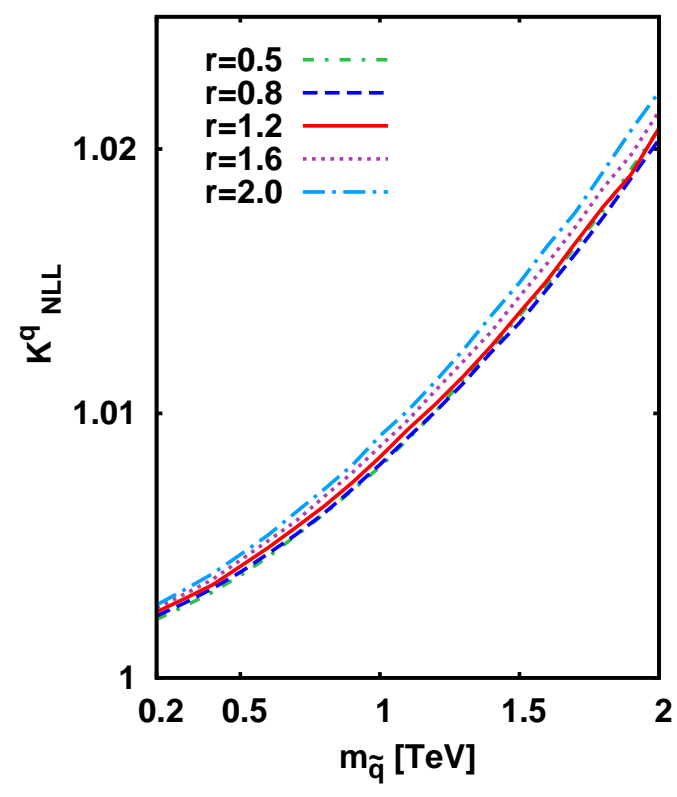

a)

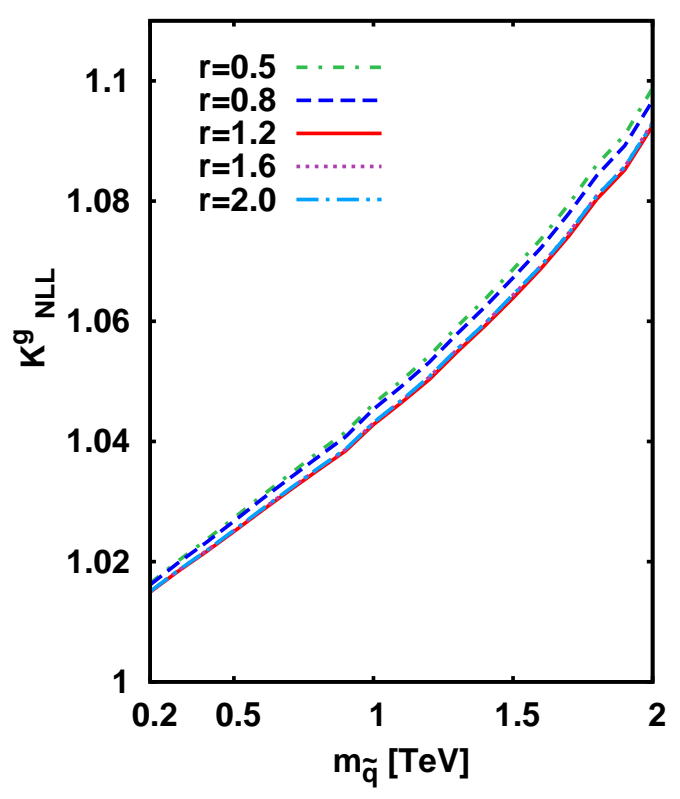

b)

Figure 10: The $K$-factor, $K_{\mathrm{NLL}}^{i}$, for the partonic channels in the hadronic process $p p \rightarrow \tilde{q} \overline{\tilde{q}}:$ (a) $q \bar{q} \rightarrow \tilde{q} \overline{\tilde{q}}$ and (b) $g g \rightarrow \tilde{q} \overline{\tilde{q}}$.

in both partonic subchannels. Such results are expected since due to larger colour factors the soft gluon corrections should be more pronounced when the particles with higher colour charges take part in the hard scattering.

We also study the relative importance of the partonic subchannels in the considered sparticle production processes. For this purpose we define the fractions $R_{\text {channel }}^{i j}=\sigma_{p p \rightarrow i j \rightarrow k l} / \sigma_{p p \rightarrow k l}$ for each total hadronic cross section, $\sigma_{p p \rightarrow k l}$, where $k l=\tilde{g} \tilde{g}$ or $k l=\tilde{q} \overline{\tilde{q}}$. The components $\sigma_{p p \rightarrow i j \rightarrow k l}$ of the hadronic cross sections come from the contributions with intermediate partons $i j$. We shall denote: $i j=q \bar{q}$ ( $q q$ in the figures) for the combined $q \bar{q}$ and $\bar{q} q$ contributions, $i j=g q$ for the combined $g q, q g, g \bar{q}$ and $\bar{q} g$ contributions, and $i j=g g$ for the $g g$ contribution. For the considered processes the $i j=g q$ contribution does not appear at the LO. In Fig. 11 and Fig. 12 we show the obtained values of $R_{\text {channel }}^{i j}$ for the $\tilde{g} \tilde{g}$ and $\tilde{q} \overline{\tilde{q}}$ production at the LHC. The results are given at the LO (Fig. 11a and Fig. 12a), at the NLO (Fig. 11b and Fig. 12b), and at the matched NLL accuracy (Fig. 11c and Fig. 12c). Clearly, the dominant contribution to the $\tilde{g} \tilde{g}$ hadroproduction comes from the $g g$ subprocess and the $\tilde{q} \overline{\tilde{q}}$ hadroproduction is dominated by the $q \bar{q}$ subprocesses except for the lower range of the squark masses where $q \bar{q}$ and $g g$ contributions are similar. The inclusion of the NLO effects leads to a relative enhancement of the $g g$ channel, and the values of $R_{\text {channel }}^{i j}$ do not change visibly between the NLO and the NLL results. The $g q$ channel appears beyond the LO and plays a minor rôle. Note that $R_{\text {channel }}^{g q}$ is negative for the gluino-pair production (see Fig. 11b and Fig. 11c), which is explained by the fact that this 


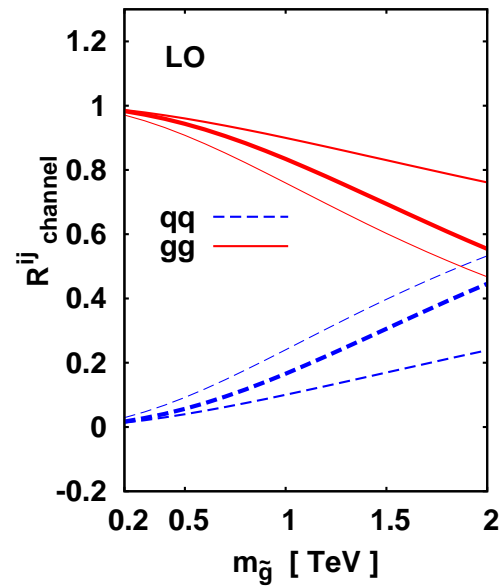

a)

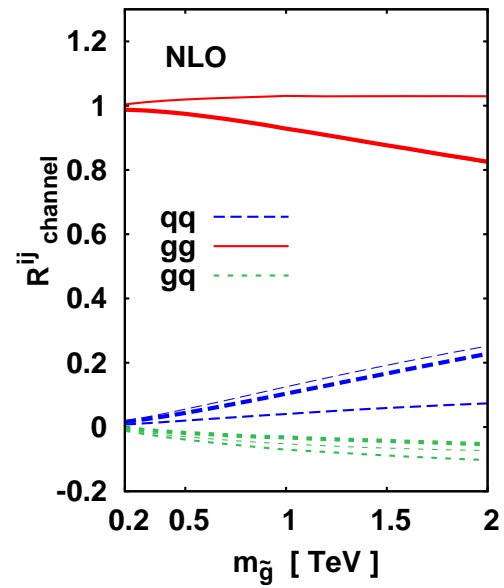

b)

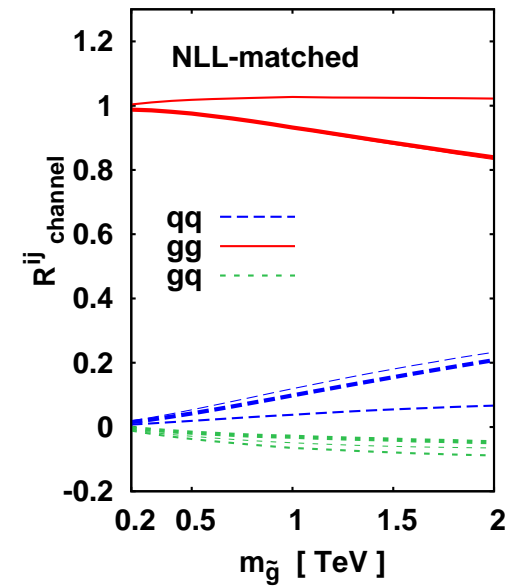

c)

Figure 11: Relative contributions of the partonic channels to the $p p \rightarrow \tilde{g} \tilde{g}$ cross section at the LO (a), NLO (b) and NLL-matched accuracy. The thick lines correspond to $r=0.5$, the medium lines to $r=1.2$, and the thin ones to $r=2.0$. The gg lines in (b) and (c) incidentally overlap for $r=0.5$ and $r=2.0$.

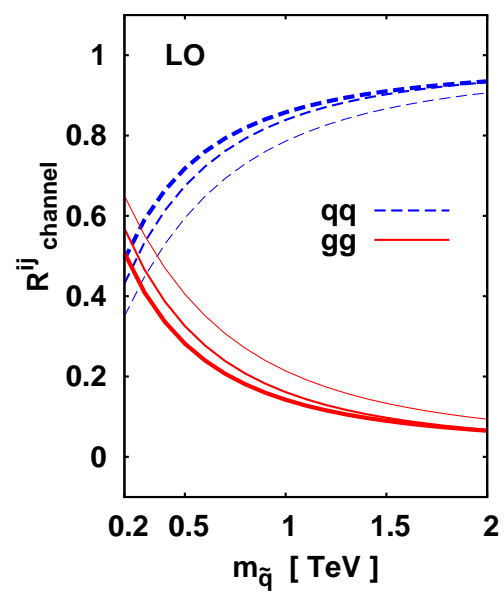

a)

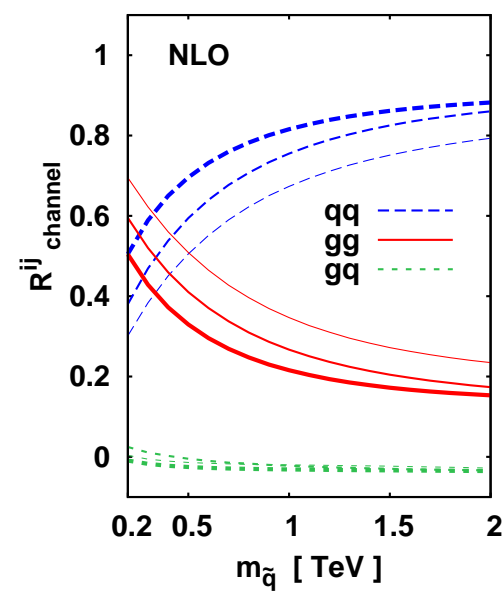

b)

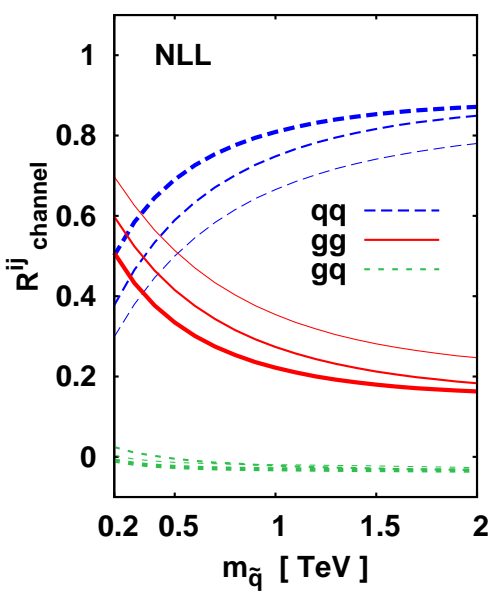

c)

Figure 12: Relative contributions of the partonic channels to the $p p \rightarrow \tilde{q} \overline{\tilde{q}}$ cross section at the LO (a), NLO (b) and NLL-matched (c) accuracy. The thick lines correspond to $r=0.5$, the medium lines to $r=1.2$, and the thin ones to $r=2.0$. 
contribution enters only beyond the LO where the simple probabilistic interpretation of the pdfs is lost.

It is interesting to check what fraction of the NLO corrections is generated by the soft gluon contributions. In order to answer this question we truncate at the NLO the expansion of the resummed hadronic cross sections $\sigma_{p p \rightarrow i j \rightarrow k l}^{(\mathrm{res})}$ mediated by the partonic subchannel $i j$ and define: $\sigma_{p p \rightarrow i j \rightarrow k l}^{(\exp )}=\left.\sigma_{p p \rightarrow i j \rightarrow k l}^{(\mathrm{res})}\right|_{(\mathrm{NLO})}$. In Fig. 13 and in Fig. 14 we plot the obtained values of the ratio, $\sigma_{p p \rightarrow i j \rightarrow k l}^{(\exp )} / \sigma_{p p \rightarrow i j \rightarrow k l}^{(\mathrm{NLO})}$, for the $\tilde{g} \tilde{g}$ production $(k l=\tilde{g} \tilde{g})$ and the $\tilde{q} \overline{\tilde{q}}(k l=\tilde{q} \tilde{\tilde{q}})$ production, respectively. It follows from the figures that the soft gluon correction provides the dominant part (more than $2 / 3$ in the studied cases) of the NLO correction in both the $q \bar{q}$ and $g g$ subchannels, and both for the $\tilde{g} \tilde{g}$ and $\tilde{q} \overline{\tilde{q}}$ production. The cusps visible in the curves in Fig. 13 originate from the NLO supersymmetric QCD correction to the $p p \rightarrow \tilde{g} \tilde{g}$ cross section. In more detail, the stop-top loop contribution to the gluino self-energy exhibits the singular behaviour in vicinity of threshold for the decay of gluino into the top quark and its superpartner, the stop.

Finally, we analyse the effect of soft gluon resummation in the partonic colour channels. Recall that the soft gluon corrections at threshold do not lead to mixing of the $s$-channel colour representations. Let us denote by $\sigma_{p p \rightarrow i j \rightarrow k l ; I}^{(\mathrm{LO})}$ the contribution to the $p p \rightarrow k l$ cross section at the LO coming from the partonic channels $i j$ in the $s$-channel colour representation $I$, and by $\sigma_{p p \rightarrow i j \rightarrow k l ; I}^{\text {(res) }}$ the analogous contribution to the resummed hadronic cross section (not matched to the NLO). Furthermore, we define ratios

$$
R_{\mathrm{color}}^{I}=\frac{\sigma_{p p \rightarrow i j \rightarrow k l ; I}}{\sum_{I} \sigma_{p p \rightarrow i j \rightarrow k l ; I}}
$$

that correspond to the relative contribution of the colour representation $I$ to the partonic channel $i j$ of the hadronic cross section (we suppress here the indices of the initial, intermediate and final state particles in $R_{\text {color }}^{I}$ ). In Fig. 15 and in Fig. 16 we show the ratios $R_{\text {color }}^{I}$ for $\tilde{g} \tilde{g}$ and $\tilde{q} \overline{\tilde{q}}$ production, respectively, both for the LO cross sections and the resummed cross sections with $r=1.2$. In general we find that the dependence of $R_{\text {color }}^{I}$ on the mass of produced particle is weak. We find that the colour singlet $s$-channel representation dominates in the $q \bar{q}$ channel of $p p \rightarrow \tilde{q} \overline{\tilde{q}}$, and the colour octet $s$-channel representation dominates in the $q \bar{q}$ channel of $p p \rightarrow \tilde{g} \tilde{g}$ and in the $g g$ channel of $p p \rightarrow \tilde{q} \tilde{\tilde{q}}$. In all these channels the soft gluon effects only weakly affect $R_{\text {color }}^{I}$. In the case of the $g g$ channel of $p p \rightarrow \tilde{g} \tilde{g}$, the pattern is more interesting. We observe the following hierarchy of the contributions from the three colour channels, $R_{\text {color }}^{\mathbf{2 7}}>R_{\text {color }}^{\mathbf{8}}>R_{\text {color }}^{\mathbf{1}}$. The largest contribution coming from the $\mathbf{2 7} s$-channel representation is most strongly enhanced due to soft gluon effects.

In Fig. 17 and Fig. 18 we show the effect of soft gluon corrections on the cross sections in partonic-colour channels for the $\tilde{g} \tilde{g}$ and $\tilde{q} \tilde{\tilde{q}}$ production, respectively. We plot the $K$-factors, $K=$ $\sigma_{I}^{(\mathrm{res})} / \sigma_{I}^{(\mathrm{LO})} \equiv \sigma_{i j \rightarrow k l, I}^{(\mathrm{res})} / \sigma_{i j \rightarrow k l, I}^{(0)}$ in these channels. The pattern is quite clear: the enhancement from soft gluon resummation for the $g g$ partonic channels is strong and steeply rising with mass 


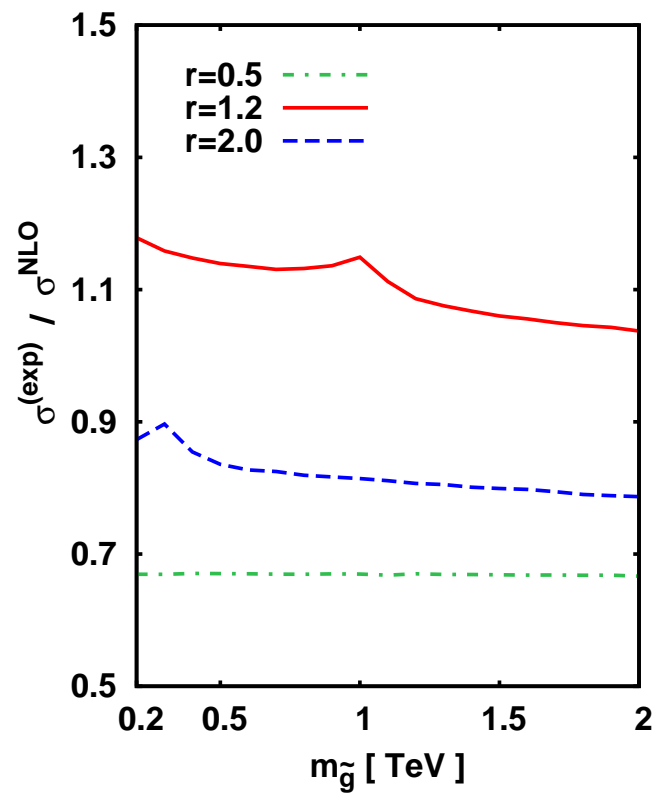

a)

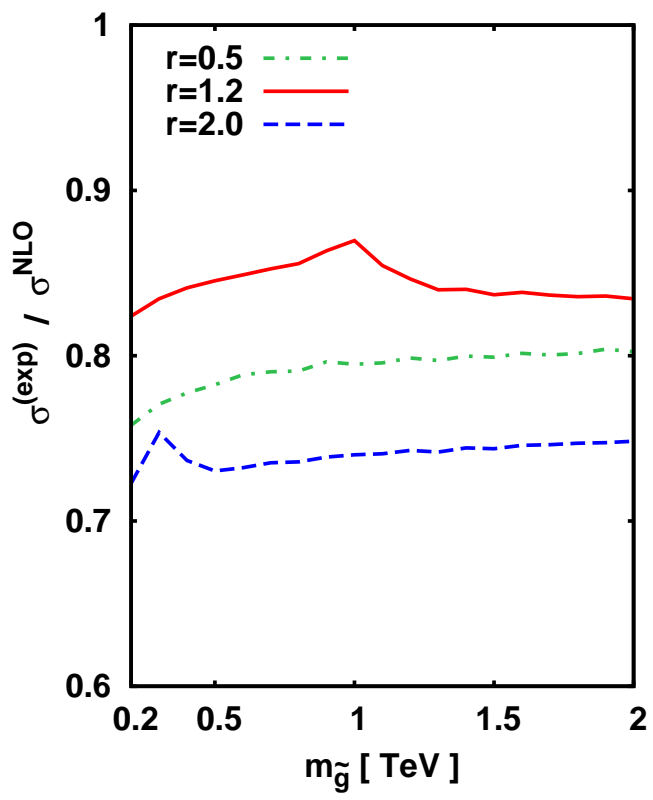

b)

Figure 13: The fraction of the NLO correction exhausted by the soft gluon contribution, $\sigma^{(\exp )} / \sigma_{(\mathrm{NLO})}$, for $(a) q \bar{q} \rightarrow \tilde{g} \tilde{g}$ and $(b) g g \rightarrow \tilde{g} \tilde{g} ; r=m_{\tilde{g}} / m_{\tilde{q}}$.

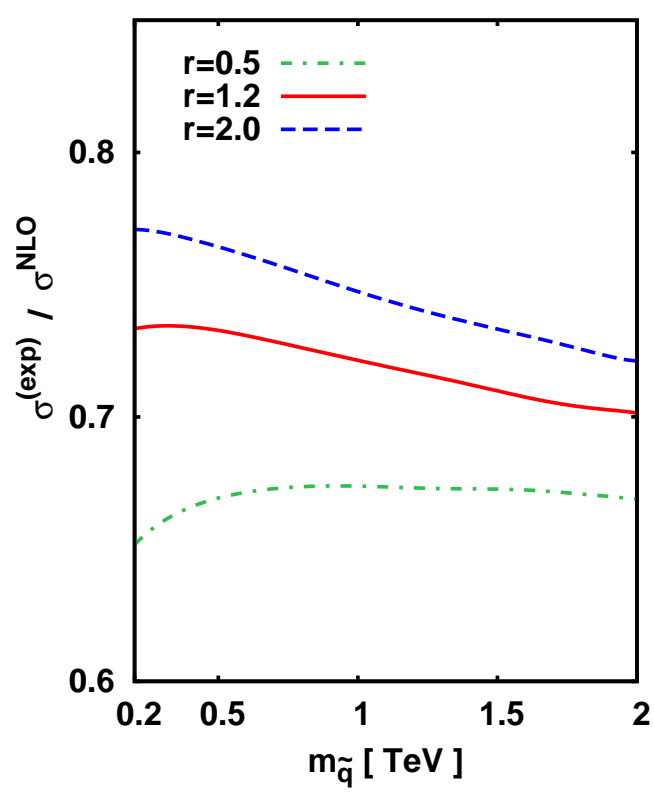

a)

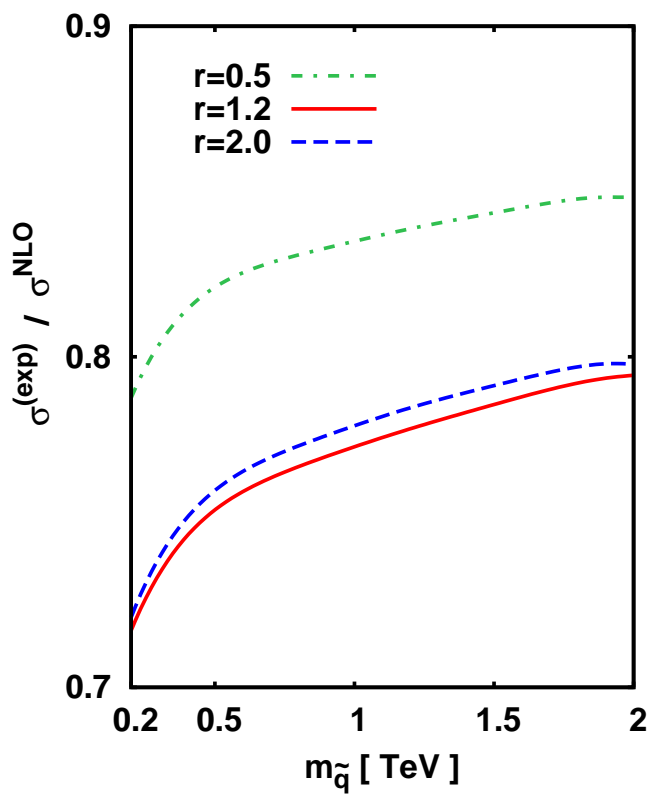

b)

Figure 14: The fraction of the NLO correction exhausted by the soft gluon contribution, $\sigma^{(\exp )} / \sigma_{\mathrm{NLO}}$, for $(a) q \bar{q} \rightarrow \tilde{q} \overline{\tilde{q}}$ and $(b) g g \rightarrow \tilde{q} \overline{\tilde{q}} ; r=m_{\tilde{g}} / m_{\tilde{q}}$. 


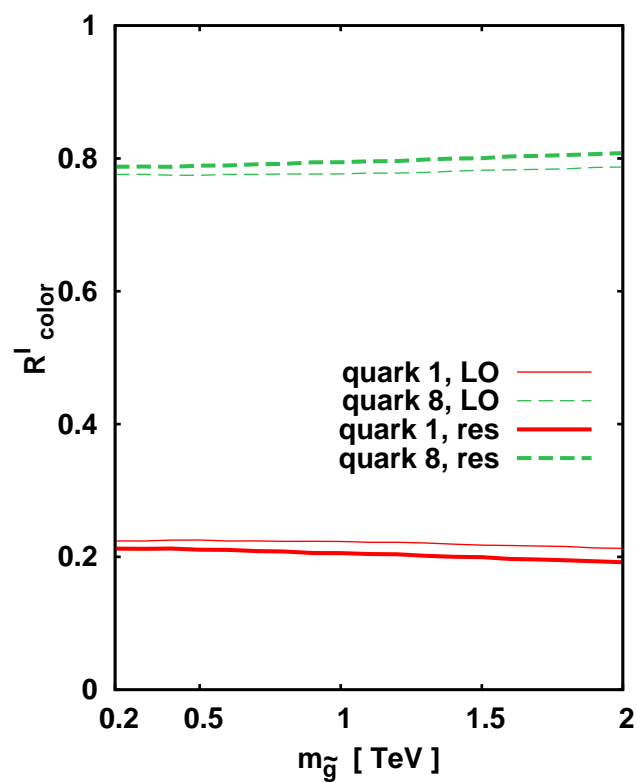

a)

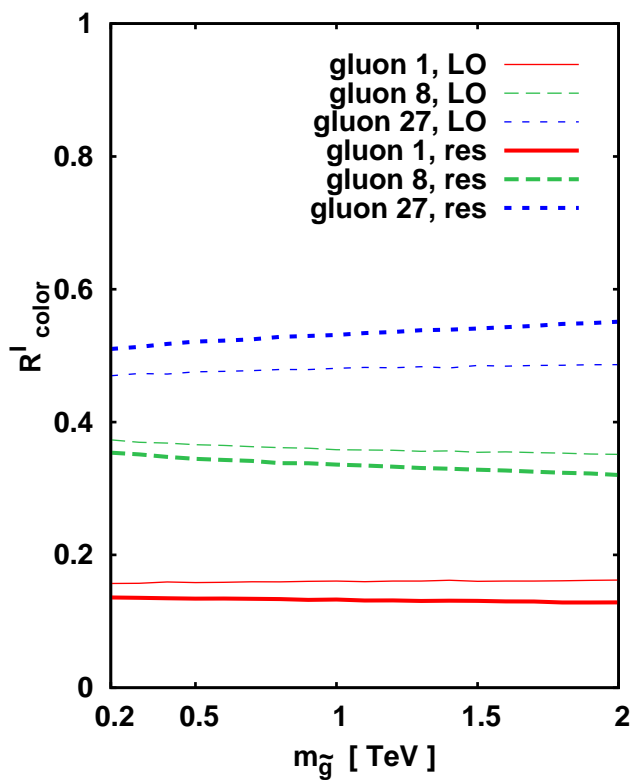

b)

Figure 15: The decomposition of the cross section for the $\tilde{g} \tilde{g}$ hadroproduction into the partonic colour channels, $R_{\text {color }}^{I}$, for: (a) $q \bar{q} \rightarrow \tilde{g} \tilde{g}$ and (b) $g g \rightarrow \tilde{g} \tilde{g} ; r=1.2$. The thick lines correspond to the resummed cross sections and the thin ones to the $L O$ cross sections.

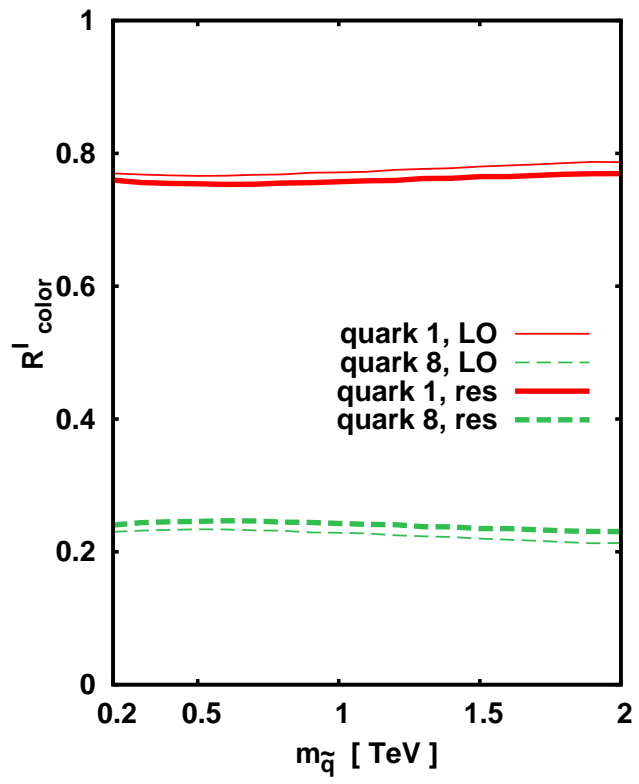

a)

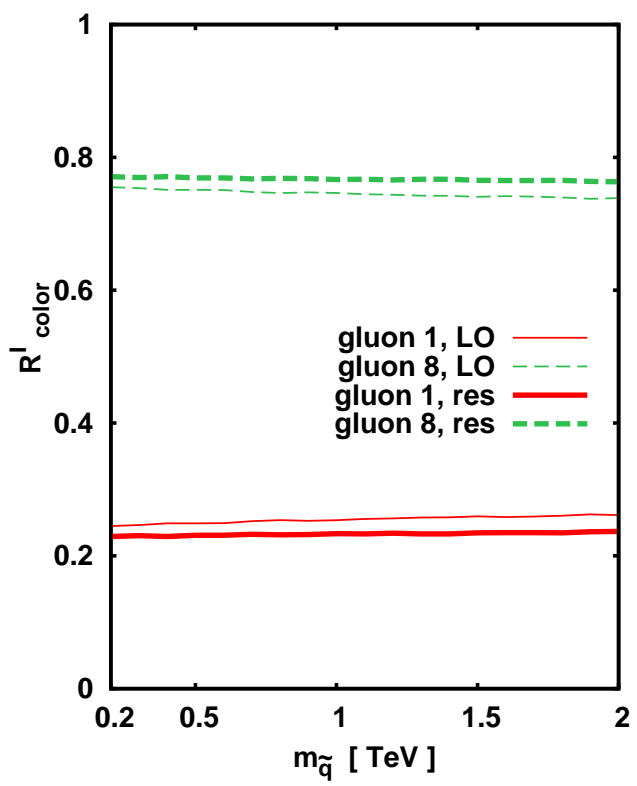

b)

Figure 16: The decomposition of the cross section for the $\tilde{q} \overline{\tilde{q}}$ hadroproduction into the partonic colour channels, $R_{\text {color }}^{I}$, for: (a) $q \bar{q} \rightarrow \tilde{q} \overline{\tilde{q}}$ and (b) $g g \rightarrow \tilde{q} \overline{\tilde{q}} ; r=1.2$. The thick lines correspond to the NLL cross sections and the thin ones to the LO cross sections. 


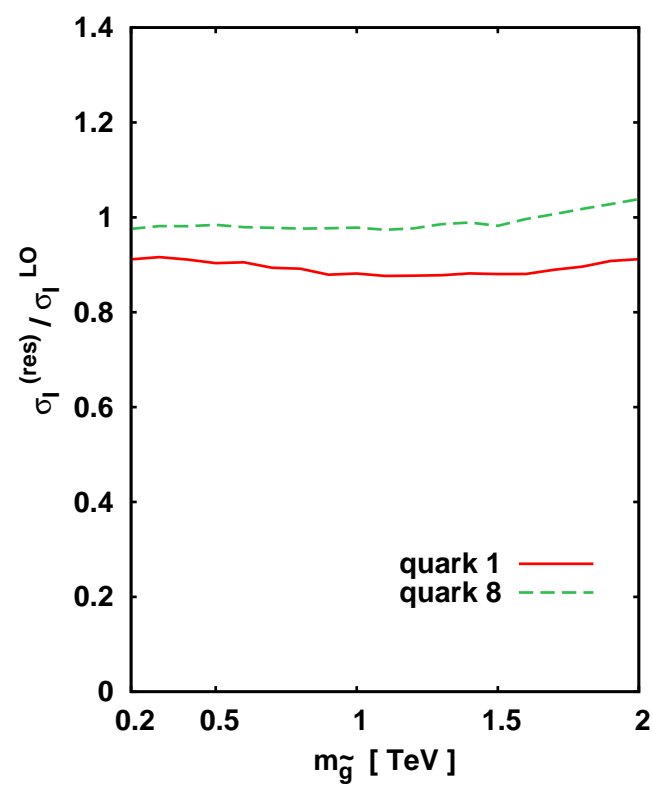

a)

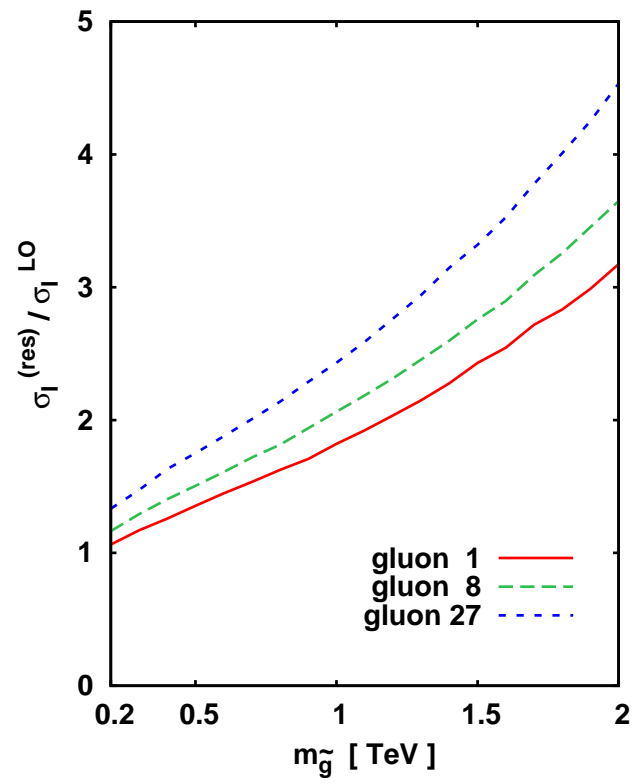

b)

Figure 17: The $K$-factors, $K=\sigma_{I}^{(\mathrm{res})} / \sigma^{(\mathrm{LO})}$, for partonic colour channels: (a) $q \bar{q} \rightarrow \tilde{g} \tilde{g}$ and (b) $g g \rightarrow \tilde{g} \tilde{g} ; r=1.2$. The dimensions of the $s$-channel colour representations are indicated in the legend.

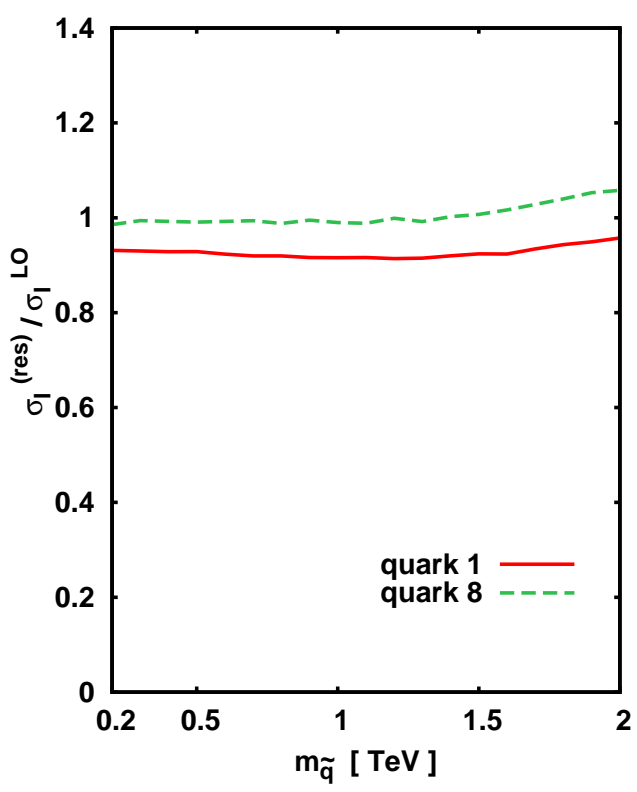

a)

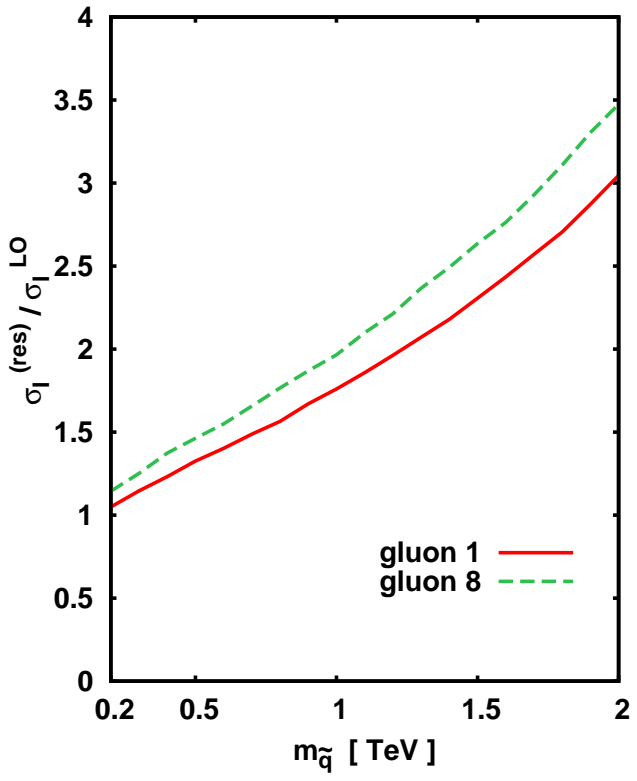

b)

Figure 18: The $K$-factors, $K=\sigma_{I}^{(\mathrm{res})} / \sigma^{(\mathrm{LO})}$, for partonic colour channels: $q \bar{q} \rightarrow \tilde{q} \overline{\tilde{q}}$ and $(b)$ $g g \rightarrow \tilde{q} \overline{\tilde{q}} ; r=1.2$. The dimensions of the $s$-channel colour representations are indicated in the legend. 
of the produced particles. This is not the case for the $q \bar{q}$ channel, where the soft gluons effects are small and weakly depending on the mass of the produced particles. Note that the $K$-factors in the $q \bar{q}$ channels are smaller than 1 for most values of the masses. In these channels, the size of the enhancement coming from soft gluon emission does not sufficiently compensate the suppresion due to changing from the LO to the NLO approximation of the pdfs and running $\alpha_{\mathrm{s}}$. For all considered channels we observe larger soft gluon enhancement for higher $s$-channel colour representations.

\section{Conclusions}

In this work we have studied the effect of soft gluon emission on the production of gluino-gluino and squark-antiquark pairs in the proton-proton collisions at the LHC. A detailed description of the derivation of the soft anomalous dimension matrices for the $q \bar{q} \rightarrow \tilde{g} \tilde{g}$ and $g g \rightarrow \tilde{g} \tilde{g}$ scattering processes [13] has been given. It should be stressed that these matrices govern the soft non-collinear gluon radiation in the hadronic pair production of any heavy particles in the colour octet representation, like for instance the supersymmetric heavy colour scalar particles considered in [44]. In the threshold limit the obtained soft anomalous dimension matrices become diagonal in the $s$-channel colour basis. The diagonal elements of the soft matrices have been found to correspond to values of the quadratic Casimir operators of the SU(3) group for the outgoing pair of heavy particles. This observation confirms simple physical picture of the direct dependence of the soft gluon radiation at threshold on the total colour charge of the final state.

The NLL resummation of the soft gluon corrections to the hadroproduction processes, $p p \rightarrow$ $\tilde{q} \overline{\tilde{q}}$ and $p p \rightarrow \tilde{g} \tilde{g}$ at the LHC has been explicitly performed for the values of masses of the produced particles between $0.2 \mathrm{TeV}$ and $2 \mathrm{TeV}$ and for the gluino-to-squark mass ratio $0.5<r<2$. The obtained results have been matched to the corresponding cross sections computed at the NLO accuracy. We have determined the NLO $K$-factors, $K_{\mathrm{NLL}}$, for the squark-antisquark and gluino pair-production for these processes and studied their scale dependence. Futhermore, we have investigated the dependence of the NLL $K$-factors on the choice of the parton distribution functions. Additionally, the effect of the leading Coulomb corrections on the considered hadronic cross sections has been discussed. We have also analysed the partonic channel decomposition of the $p p \rightarrow \tilde{g} \tilde{g}$ and $p p \rightarrow \tilde{q} \overline{\tilde{q}}$ total cross sections. In particular, we have studied the effect of the NLO and NLL corrections on the total cross sections in the partonic channels. Finally, we have decomposed the LO and NLL hadronic cross sections for the partonic subchannels in the colour basis and observed that the soft gluon enhancement grows with the the total colour charge of the pair of the produced particles.

Acknowledgments A. K. thanks W. Beenakker, S. Brensing, M. Krämer, E. Laenen and I. Niessen for many discussions. L.M. is grateful to J. Bartels for stimulating conversations. The 
work of A.K. is supported by the Initiative and Networking Fund of the Helmholtz Association, contract HA-101 ("Physics at the Terascale"). L. M. acknowledges the DFG grant No. SFB 676 and of the Polish Ministry of Education grant No. N202 249235.

\section{A Mellin-moment transforms of the leading-order cross sections}

We define

$$
\begin{aligned}
J_{N} & \equiv \int_{0}^{1} d z \frac{z^{N+1}}{1+\left(\frac{1-r^{2}}{2 r^{2}}\right) z} \log \left(\frac{2 r^{2}(1+\sqrt{1-z})+\left(1-r^{2}\right) z}{2 r^{2}(1-\sqrt{1-z})+\left(1-r^{2}\right) z}\right) \\
B_{N} & \equiv \beta(N+1,1 / 2) \\
G_{N}^{(1)} & \equiv{ }_{2} F_{1}\left(1,1 / 2, N+5 / 2,\left(\frac{r^{2}-1}{r^{2}+1}\right)^{2}\right), \\
G_{N}^{(2)} & \equiv{ }_{2} F_{1}\left(1, N+1, N+5 / 2,-\frac{\left(r^{2}-1\right)^{2}}{4 r^{2}}\right),
\end{aligned}
$$

where $r=m_{\tilde{g}} / m_{\tilde{q}}$. With these definitions, the LO cross sections in $N$-space read

$$
\begin{aligned}
& \tilde{\sigma}_{q_{i} \bar{q}_{j} \rightarrow \tilde{q} \tilde{\tilde{q}}, \mathbf{1}}^{(0)}=\frac{\alpha_{\mathrm{s}}^{2} \pi B_{N}}{m_{\tilde{q}}^{2}} \frac{8}{81\left(2 N^{3}+9 N^{2}+13 N+6\right)\left(r^{2}+1\right)} \\
& \times\left[-\left(r^{2}+1\right) N(2 N+3)+2\left(r^{2} N+N+2\right)(N+1) G_{N}^{(1)}+\left(r^{2}+1\right)\left(N^{2}+3 N+2\right) G_{N}^{(2)}\right], \\
& \tilde{\sigma}_{q_{i} \bar{q}_{j} \rightarrow \tilde{q} \tilde{\tilde{q}}, \mathbf{8}}^{(0)}=\frac{\alpha_{\mathrm{s}}{ }^{2} \pi B_{N}}{m_{\tilde{q}}^{2}}\left[\frac{\delta_{i j} n_{f}}{9\left(4 N^{2}+16 N+15\right)}\right. \\
& \left.+\frac{2 \delta_{i j}\left(\left(r^{2}+1\right)\left(3 r^{2}+N\left(2 r^{2}-1\right)-2\right)-2 r^{4}(N+1) G_{N}^{(1)}\right)}{27\left(2 N^{3}+13 N^{2}+27 N+18\right)\left(r^{4}-1\right)}\right]+\frac{1}{8} \hat{\sigma}_{q \bar{q} \rightarrow \tilde{q} \tilde{\tilde{q}}, \mathbf{1}}^{(0)}, \\
& \tilde{\sigma}_{g g \rightarrow \tilde{q} \overline{\tilde{q}}, \mathbf{1}}^{(0)}=\frac{\alpha_{\mathrm{s}}^{2} \pi B_{N}}{m_{\tilde{q}}^{2}} \frac{\left(N^{2}+3 N+4\right) n_{f}}{96\left(2 N^{3}+13 N^{2}+27 N+18\right)}, \\
& \tilde{\sigma}_{g g \rightarrow \tilde{q} \tilde{\tilde{q}}, \mathbf{8}}^{(0)}=\frac{\alpha_{\mathrm{s}}{ }^{2} \pi B_{N}}{m_{\tilde{q}}^{2}} \frac{\left(5 N^{3}+32 N^{2}+71 N+68\right) n_{f}}{96\left(4 N^{4}+36 N^{3}+119 N^{2}+171 N+90\right)}, \\
& \tilde{\sigma}_{q \bar{q} \rightarrow \tilde{g} \tilde{g}, \mathbf{1}}^{(0)}=-\frac{\alpha_{\mathrm{s}}{ }^{2} \pi B_{N}}{m_{\tilde{g}}^{2}} \frac{1}{54\left(2 N^{2}+7 N+6\right)\left(r^{2}+1\right)}\left[\left(r^{2}+1\right)(-8 N-12)\right. \\
& \left.+(8 N+8) G_{N}^{(1)}+2\left(r^{2}+1\right)(N+2) G_{N}^{(2)}+\left(r^{2}+1\right)\left(2 N^{2}+7 N+6\right) \frac{J_{N}}{B_{N}}\right], \\
& \tilde{\sigma}_{q \bar{q} \rightarrow \tilde{g} \tilde{g}, \mathbf{8}}^{(0)}=\frac{\alpha_{\mathrm{s}}{ }^{2} \pi B_{N}}{m_{\tilde{g}}{ }^{2}} \frac{1}{27\left(4 N^{4}+36 N^{3}+119 N^{2}+171 N+90\right)\left(r^{4}-1\right)} \\
& \times\left[2\left(r^{2}+1\right)\left(\left(r^{2}-1\right) N^{3}-\left(43-25 r^{2}\right) N^{2}-\left(162-99 r^{2}\right) N+108 r^{2}-153\right)\right. \\
& +4\left(2 N\left(r^{2}-1\right)+6 r^{2}+3\right)\left(2 N^{2}+7 N+5\right) G_{N}^{(1)} \\
& -7\left(r^{4}-1\right)\left(2 N^{3}+15 N^{2}+37 N+30\right) G_{N}^{(2)} \\
& \left.+\left(r^{4}-1\right)\left(4 N^{4}+36 N^{3}+119 N^{2}+171 N+90\right) \frac{J_{N}}{B_{N}}\right],
\end{aligned}
$$




$$
\begin{aligned}
\tilde{\sigma}_{\text {sym }} & =\frac{\alpha_{\mathrm{s}}{ }^{2} \pi B_{N}}{m_{\tilde{g}}^{2}} \frac{9\left(N^{3}+9 N^{2}+20 N+14\right)}{64\left(2 N^{4}+15 N^{3}+40 N^{2}+45 N+18\right)}, \\
\tilde{\sigma}_{\text {asym }} & =\frac{\alpha_{\mathrm{s}}{ }^{2} \pi B_{N}}{m_{\tilde{g}}^{2}} \frac{9\left(N^{3}+11 N^{2}+30 N+26\right)}{64\left(4 N^{5}+40 N^{4}+155 N^{3}+290 N^{2}+261 N+90\right)},
\end{aligned}
$$

where we put $\hat{\alpha}_{\mathrm{s}}=\alpha_{\mathrm{s}}$. For the numerical evaluation of $J_{N}$ we separately use two forms of its expansion

$$
J_{N}=\frac{2 r^{2}}{1+r^{2}} \sum_{m=0}^{\infty}\left(\frac{1-r^{2}}{1+r^{2}}\right)^{m} \sum_{k=0}^{\infty} \frac{1-\left(\frac{1-r^{2}}{1+r^{2}}\right)^{2 k+1}}{k+1 / 2} \beta(N+2, k+m+3 / 2),
$$

and

$$
\begin{aligned}
& J_{N}=\frac{2 r^{2}}{1+r^{2}} \sum_{m=0}^{\infty}\left(\frac{1-r^{2}}{1+r^{2}}\right)^{m} \frac{1}{1+m} \sum_{k=0}^{m} \frac{(-1)^{k}}{\beta(k+1, m-k+1)} \\
& \times\left[\frac{\beta(k+N+2,1 / 2)}{k+N+2}-2\left(\frac{1-r^{2}}{1+r^{2}}\right) \beta(k+N+2,3 / 2){ }_{2} F_{1}\left(1,1 / 2, k+N+7 / 2,\left(\frac{1-r^{2}}{1+r^{2}}\right)^{2}\right)\right] .
\end{aligned}
$$

\section{B LL and NLL functions}

The expressions for the resummed factors, expanded up to NLL, are

$$
\begin{aligned}
& \log \Delta_{i}\left(N, 4 m^{2}, \mu^{2}\right) \stackrel{\mathrm{NLL}}{=} g_{i}^{(1)}\left(b_{0} \alpha_{s}\left(\mu^{2}\right) \log N\right) \log N+g_{i}^{(2)}\left(b_{0} \alpha_{s}\left(\mu^{2}\right) \log N, 4 m^{2}, \mu^{2}\right), \\
& \log \Delta_{i j \rightarrow k l, I}^{(s)}\left(N, 4 m^{2}, \mu^{2}\right) \stackrel{\mathrm{NLL}}{=} h_{i j \rightarrow k l, I}^{(2)}\left(b_{0} \alpha_{s}\left(\mu^{2}\right) \log N\right)
\end{aligned}
$$

with

$$
\begin{aligned}
g_{i}^{(1)}(\lambda)= & \frac{A_{i}^{(1)}}{2 \pi b_{0} \lambda}[2 \lambda+(1-2 \lambda) \log (1-2 \lambda)], \\
g_{i}^{(2)}\left(\lambda, 4 m^{2}, \mu^{2}\right)= & -\frac{A_{i}^{(1)} \gamma_{E}}{\pi b_{0}} \log (1-2 \lambda)+\frac{A_{i}^{(1)} b_{1}}{2 \pi b_{0}^{3}}\left[2 \lambda+\log (1-2 \lambda)+\frac{1}{2} \log ^{2}(1-2 \lambda)\right] \\
& -\frac{A_{i}^{(2)}}{2 \pi^{2} b_{0}^{2}}[2 \lambda+\log (1-2 \lambda)]-\frac{A_{i}^{(1)}}{2 \pi b_{0}} \log (1-2 \lambda) \log \left(\frac{\mu^{2}}{4 m^{2}}\right), \\
h_{i j \rightarrow k l, I}^{(2)}(\lambda)= & \frac{\log (1-2 \lambda)}{2 \pi b_{0}} D_{i j \rightarrow k l, I}^{(1)},
\end{aligned}
$$

where we took $\mu=\mu_{F}=\mu_{R}$ and $b_{0}$ and $b_{1}$ are the first two coefficients of the QCD $\beta$-function,

$$
b_{0}=\frac{11 C_{A}-4 T_{R} n_{\mathrm{f}}}{12 \pi}, \quad b_{1}=\frac{17 C_{A}^{2}-10 C_{A} T_{R} n_{\mathrm{f}}-6 C_{F} T_{R} n_{\mathrm{f}}}{24 \pi^{2}} .
$$

The values of the coefficients $A_{i}^{(1)}, A_{i}^{(2)}, D_{i j \rightarrow k l, I}^{(1)}$ are defined in Section 3.1. 


\section{References}

[1] H. E. Haber and G. L. Kane, Phys. Rept. 117, 75 (1985).

[2] see e.g. M. Drell, R. Godbole and P. Roy, "Theory and Phenomenology of Sparticles", World Scientific (2004), and refs. therein.

[3] ATLAS Technical Design Report, Vol. II, ATLAS TDR 14, CERN/LHCC 99-14, 1999; CMS Physics Technical Design Report, Vol. II, CERN/LHCC 06-021, CMS TDR 8.2, 2006.

[4] P. R. Harrison and C. H. Llewellyn Smith, Nucl. Phys. B 213 (1983) 223 [Erratum-ibid. B 223 (1983) 542].

[5] S. Dawson, E. Eichten and C. Quigg, Phys. Rev. D 31, 1581 (1985).

[6] W. Beenakker, R. Höpker, M. Spira and P. M. Zerwas, Phys. Rev. Lett. 74, 2905 (1995); Z. Phys. C 69, 163 (1995); W. Beenakker, M. Krämer, T. Plehn, M. Spira and P. M. Zerwas, Nucl. Phys. B 515, 3 (1998).

[7] W. Beenakker, R. Höpker, M. Spira and P. M. Zerwas, Nucl. Phys. B 492, 51 (1997).

[8] H. Baer, V. Barger, G. Shaughnessy, H. Summy and L. t. Wang, Phys. Rev. D 75, 095010 (2007).

[9] V. M. Abazov et al. [D0 Collaboration], Phys. Lett. B 638 (2006) 119; Phys. Lett. B 660 (2008) 449.

[10] T. Aaltonen et al. [CDF Collaboration], Phys. Rev. Lett. 102 (2009) 121801.

[11] W. Hollik, E. Mirabella and M. K. Trenkel, JHEP 0902 (2009) 002; W. Hollik and E. Mirabella, JHEP 0812 (2008) 087; W. Hollik, M. Kollar and M. K. Trenkel, JHEP 0802 (2008) 018.

[12] A. T. Alan, K. Cankocak and D. A. Demir, Phys. Rev. D 75 (2007) 095002 [Erratum-ibid. D 76 (2007) 119903]; S. Bornhauser, M. Drees, H. K. Dreiner and J. S. Kim, Phys. Rev. D 76 (2007) 095020.

[13] A. Kulesza and L. Motyka, Phys. Rev. Lett. 102 (2009) 111802.

[14] U. Langenfeld and S. O. Moch, arXiv:0901.0802 [hep-ph].

[15] A. Idilbi, C. Kim and T. Mehen, arXiv:0903.3668 [hep-ph].

[16] W. Beenakker, S. Brensing, M. Krämer, A. Kulesza, E. Laenen and I. Niessen, to appear.

[17] M. Beneke, P. Falgari and C. Schwinn, to appear. 
[18] J. Botts and G. Sterman, Nucl. Phys. B 325, 62 (1989).

[19] N. Kidonakis and G. Sterman, Phys. Lett. B 387 (1996) 867; Nucl. Phys. B 505, 321 (1997).

[20] N. Kidonakis, G. Oderda and G. Sterman, Nucl. Phys. B 525, 299 (1998).

[21] N. Kidonakis, G. Oderda and G. Sterman, Nucl. Phys. B 531, 365 (1998).

[22] R. Bonciani, S. Catani, M. L. Mangano and P. Nason, Phys. Lett. B 575, 268 (2003).

[23] R. Bonciani, S. Catani, M. L. Mangano and P. Nason, Nucl. Phys. B 529, 424 (1998).

[24] S. Mert Aybat, L. J. Dixon and G. Sterman, Phys. Rev. Lett. 97, 072001 (2006); Phys. Rev. D 74, 074004 (2006).

[25] S. Moch and P. Uwer, Phys. Rev. D 78 (2008) 034003.

[26] A. Mitov, G. Sterman and I. Sung, arXiv:0903.3241 [hep-ph].

[27] S. Catani, M. L. Mangano, P. Nason and L. Trentadue, Nucl. Phys. B 478, 273 (1996).

[28] G. Sterman, Nucl. Phys. B 281 (1987) 310.

[29] L. J. Dixon, L. Magnea and G. Sterman, JHEP 0808, 022 (2008).

[30] S. Catani and L. Trentadue, Nucl. Phys. B 327 (1989) 323.

[31] J. Kodaira and L. Trentadue, Phys. Lett. B 112 (1982) 66,

[32] S. Catani, E. D'Emilio and L. Trentadue, Phys. Lett. B 211 (1988) 335.

[33] R. Mertig, M. Bohm and A. Denner, Comput. Phys. Commun. 64 (1991) 345; http://www.feyncalc.org/

[34] T. van Ritbergen, A. N. Schellekens and J. A. M. Vermaseren, Int. J. Mod. Phys. A 14 (1999) 41.

[35] J. A. M. Vermaseren, arXiv:math-ph/0010025.

[36] E. Laenen, G. Sterman and W. Vogelsang, Phys. Rev. D 63 (2001) 114018.

[37] V. S. Fadin, V. A. Khoze and T. Sjostrand, Z. Phys. C 48 (1990) 613;

S. Catani, M. L. Mangano, P. Nason and L. Trentadue, Phys. Lett. B 378 (1996) 329.

[38] K. Hagiwara, Y. Sumino and H. Yokoya, Phys. Lett. B 666 (2008) 71;

Y. Kiyo, J. H. Kuhn, S. Moch, M. Steinhauser and P. Uwer, Eur. Phys. J. C 60 (2009) 375.

[39] A. Kulesza, G. Sterman and W. Vogelsang, Phys. Rev. D 66 (2002) 014011. 
[40] A. Kulesza, G. Sterman and W. Vogelsang, Phys. Rev. D 69 (2004) 014012.

[41] W. Beenakker, R. Höpker and M. Spira, arXiv:hep-ph/9611232; http://www.ph.ed.ac.uk/ tplehn/prospino/

[42] W. K. Tung, H. L. Lai, A. Belyaev, J. Pumplin, D. Stump and C. P. Yuan, JHEP 0702, 053 (2007).

[43] A. D. Martin, W. J. Stirling, R. S. Thorne and G. Watt, arXiv:0901.0002 [hep-ph].

[44] S. Y. Choi, M. Drees, J. Kalinowski, J. M. Kim, E. Popenda and P. M. Zerwas, Phys. Lett. B 672 (2009) 246. 